University of Nebraska - Lincoln

DigitalCommons@University of Nebraska - Lincoln

Investigations of the Ichthyofauna of

Nicaraguan Lakes

Papers in the Biological Sciences

1976

Peces de la cuenca de los grandes lagos de Nicaragua

Ignacio Astorqui

Colegio Centro America

Follow this and additional works at: https://digitalcommons.unl.edu/ichthynicar

Part of the Aquaculture and Fisheries Commons

Astorqui, Ignacio, "Peces de la cuenca de los grandes lagos de Nicaragua" (1976). Investigations of the Ichthyofauna of Nicaraguan Lakes. 14.

https://digitalcommons.unl.edu/ichthynicar/14

This Article is brought to you for free and open access by the Papers in the Biological Sciences at DigitalCommons@University of Nebraska - Lincoln. It has been accepted for inclusion in Investigations of the Ichthyofauna of Nicaraguan Lakes by an authorized administrator of DigitalCommons@University of Nebraska -

Lincoln. 


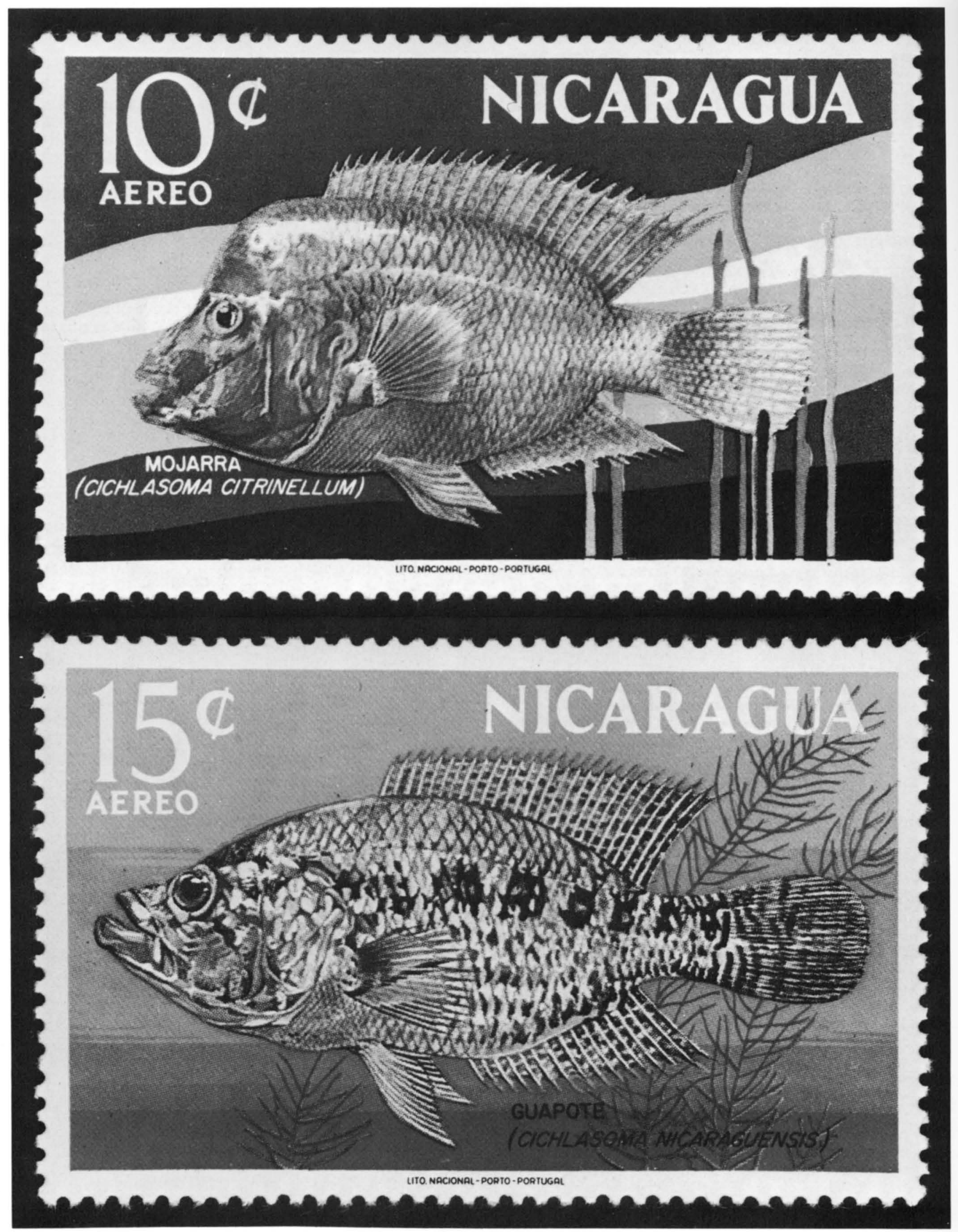

Mojarras and guapotes, the most important food fishes of inland Nicaragua, tipify the bony fishes and fisheries of Nicaraguan lakes. They are among several freshwater species that have been memorialized in a series of Nicaraguan stamps. 
Rev. Biol. Trop., 19(1, 2): 7-57, 1971

\title{
Peces de la cuenca de los grandes lagos de Nicaragua
}

\author{
por \\ Ignacio Astorqui, S. J." \\ (Recibido para su publicación el 3 de marzo de 1967)
}

El presente trabajo se basa en una colección de peces hecha en Nicaragua por el Profesor Luis R. Rivas y el autor desde el 11 de Junio hasta el 14 de Julio de 1960. El trabajo se limita a la cuenca de los Grandes Lagos y ríos tributarios (Fig. 1). La mayor parte del material procede de esta zona y no pretende ser un trabajo ictiológico definitivo acerca de Nicaragua.

Aspectos de la paleografía de Centroamérica, el origen de la fauna de los lagos y la historia de la ictiología de Nicaragua se encuentran en un trabajo an. terior (AsTORQui, 1).

\section{LOCALIDADES DE RECOLECCION}

El Lago Nicaragua tiene unos $177 \mathrm{~km}$ de largo y unos 65 de ancho y es generalmente poco profundo, siendo la máxima de unos $70 \mathrm{~m}$. El Lago Managua es más pequeño, de unos $65 \mathrm{~km}$ de largo y unos 40 de ancho con una profundidad de aproximadamente $30 \mathrm{~m}$. Durante el tiempo de nuestra expedición el nivel de las aguas era excepcionalmente bajo en ambos lagos. Las aguas estaban fangosas con una cantidad considerable de materia orgánica en suspensión. Las localidades en las que recolectamos fueron numeradas $y$ distribuidas lo más regularmente posible a lo largo de las costas de los lagos y ríos tributarios (Fig. 1), y se anotan a continuación

Localidad 1. Canales entre las "Isletas", como a $5 \mathrm{~km}$ SE de Granada. Agua fangosa y de color marrón verdoso; temperatura $28.5 \mathrm{C}$.

LOCALIDAD 2. Riachuelo que desemboca en el Lago Nicaragua, cruzando el camino de Granada a las Isletas, como a $2 \mathrm{~km} \mathrm{~S}$ de Granada; agua limpia, fondo fangoso.

Localidad 3. Tapetate, boca de una pequeña corriente en la costa del Lago Nica. ragua, como a $2 \mathrm{~km} \mathrm{~N}$ de Granada; fondo barro $y$ arena.

* Colegio Centro América, Apartado 2419, Managua, D. N., Nicaragua. 
Localtoad 4. Desembocadura de la laguna de Tisma, en el Paso de Panaloya, $21 \mathrm{~km}$ de Granada; agua sucia y muy caliente, 29 a $30 \mathrm{C}$; fondo de barro; abundante ve. Eetación en las orillas.

Localidad 5. Río Manare, al final de la carretera de Nandaime como a unos $20 \mathrm{~km} \mathrm{~S}$ de Granada; agua limpia; temperatura $28.4 \mathrm{C}$; fondo de arena y barro; abun. dante vegetación en las orillas.

Lochlidad 6. Laguna de Apoyo, como a $6 \mathrm{~km} 0$ de Granada; agua muy limpia, de gusto salobre; temperatura $28 \mathrm{C}$; fondo de arena, piedra y rocas; sin vegetación.

Localidad 7. Costa $\mathrm{E}$ del Lago de Masaya a unos $18 \mathrm{~km} O$ de Granada; agua turbia; costa rocosa; fondo de arena, rocas y barro.

Localidad 12. Rio Tisla junto a la carretera Panamericana, $18 \mathrm{~km} \mathrm{SE} \mathrm{de} \mathrm{Ri.}$ vas; fondo de arena y barro; temperatura del agua 26.2 a $28.3 \mathrm{C}$; sin vegetación acuática. Localidad 13. Costa NO de la isla Momotombito, Lago Managua; fondo de arena volcánica, piedras y grandes rocas; agua sucia y sin vegetación; temperatura $27 \mathrm{C}$

Locai.idad 14. Costa sur del Lago Managua, en Mateare, $18 \mathrm{~km}$ ONO de Ma. nagua; agua turbia; fondo de barro y arena volcánica; sin vegetación acuática; zonas de zacate a lo largo de la orilla.

LoCalidad 15. Riachuelo junto a la carretera de Managua o Tipitapa, $18 \mathrm{~km} \mathrm{E}$ de Managua; agua clara; corriente fuerte aun en las pozas; fondo de barro, arena y rocas.

Localidad 16. Desagüe del Lago Managua, Río Tipitapa, $1 \mathrm{~km} O$ de Tipitapa; fondo de barro, costas bajas; vegetación acuática ausente; agua turbia y sin corriente; ten. peratura $29.1 \mathrm{C}$.

Localidad 17. Río Ochomogo, junto a la carretera Panamericana, $14 \mathrm{~km} \mathrm{~S}$ de Nandaime; agua turbia; temperatura $27 \mathrm{C}$; corriente de suave a fuerte; fondo de rocas, piedras grandes, barro y arena; sin vegetación acuática.

Localidad 24. Río Maderas, en Las Maderas junto a la carretera Panamericana, $28 \mathrm{~km} \mathrm{~N}$ de Tipitapa; agua algo turbia; corriente lenta en las pozas entre los rápidos; fon. do barro, arena y rocas; rocas y fango en las orillas; sin vegetación acuática.

Locai.idad 25. Lago Managua, en San Francisco del Carnicero; ejemplar de Cichlasoma managuense coleccionado por Rubén Camacho.

Localidad 28. Río Mayales, $2 \mathrm{~km} \mathrm{E}$ de Juigalpa, en la carreterz de Managua al Rama; agua algo turbia; temperatura $26.5 \mathrm{C}$; corriente lenta; fondo de barro, arena y rocas.

I.ocaitidad 29. Río Isala, $22 \mathrm{~km}$ E del río Tecolostote, en 12 carretera de Ma. nagua a Juigalpa, $35 \mathrm{~km} O$ de Juigalpa; agua algo turbia; temperatura $26.5 \mathrm{C}$; fondo de barro, arena y rocas.

Localidad 30. Río Tecolostote, $25 \mathrm{~km}$ NO de Juigalpa, en la carretera de Ma. nagua a Juigalpa; orillas con abundante vegetación que se adentra en el agua; grandes masas de Spirogyra; fondo de arena, barro y rocas.

Localidad 31. Río Malacatoya, en Las Banderas, junto a la carretera de Ma. nagua a Juigalpa; agua turbia; temperatura $28.5 \mathrm{C}$; fondo de arena, barro y rocas; ori llas fangosas o rocosas.

LOCALIDAD 35. Río Ochomogo, en la carretera $\sin$ pavimentar, $3 \mathrm{~km}$ al $\mathrm{S}$ de Nandaime; agua algo turbia; temperatura $25.3 \mathrm{C}$; fondo barro y arena; corriente de suave a fuerte; sin vegetación acuática.

Localid ad 36. Río Osayo, junto a la carretera Panamericana, a $9 \mathrm{~km}$ de la frontera de Costa Rica; agua clara; temperatura $25.5 \mathrm{C}$; poca corriente; fondo y orillas de arearava y guijarros; fondo y orillas sin vegetación.

\section{METODO DE MEDIR Y CONTAR}

Mientras no se indique otra cosa, las medidas son en mm y los métodos empleados son los siguientes: longitud estándard es la distancia desde la punta del morro hasta la mitad de la base de la aleta caudal; las longitudes pre. 
ASTORQUi: PECES DE LA CUENCA dE los LAgOS DE NICARAGUA

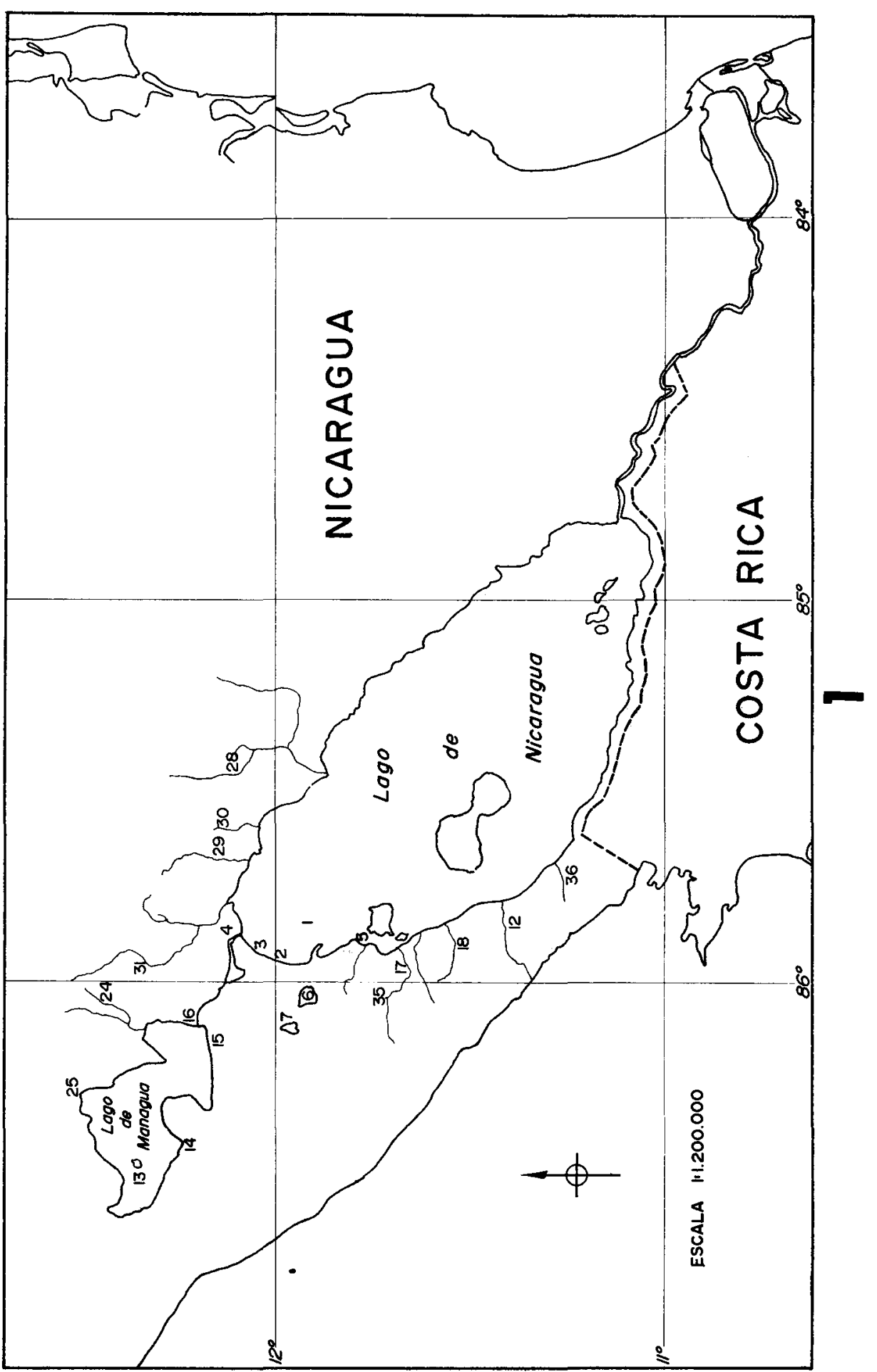


dorsal, preanal y prepélvica son las distancias desde la punta del morro hasta el origen de estas aletas; la longitud de la cabeza es la distancia desde el extremo del morro hasta la parte más distante del margen membranoso del opérculo o agalla; la longitud del morro es la distancia desde su extremo hasta el punto más cercano del borde carnoso de la órbita; el diámetro orbital se tomó entre los bordes carnosos en una dirección horizontal; el ancho interorbital se tomó en su parte más estrecha entre los bordes carnosos; la longitud del maxilar fue tomada desde el extremo del morro hasta el extremo posterior de esta estructura; la longitud de las aletas se tomó estando ellas plegadas. Cuando en lugar de longitud se indica altura, ésta se mide desde el origen de la aleta correspondiente hasta el extremo de la espina o radio más largo.

Se contaror todas las espinas y radios $(D .=$ aleta dorsal; $A .=$ aleta anal; y Pct.= aleta pectoral). Las escamas se contaron a lo largo de la línea lateral comenzando por la escama inmediatamente encima del comienzo de la ranura del opérculo o agalla y terminando en la base de la aleta caudal. Las escamas de las mejillas se calcularon como el número de filas en una línea diagonal desde la órbita hasta el ángulo del preopérculo. Los filamentos branquiales se contaron en el primer arco incluyendo el filamento en el ángulo con los del brazo inferior.

Todas las proporciones se dan en tantos por mil de la longitud estándard; los promedios se indican entre paréntesis.

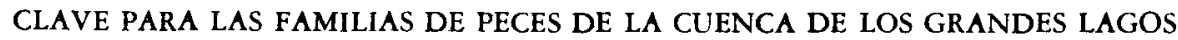

Para la fácil interpretación de la clave adjunta, téngase en cuenta que el apartado 1. estará dividido en $2 \mathrm{a}$ y $2 \mathrm{~b}$; el por cjemplo $6 \mathrm{a}$ en $7 \mathrm{a}$ y $7 \mathrm{~b}$ etc.

1a. Aberturas branquiales que no confluyen formando una sola abertura ventral.

2a. Cinco aberturas branquiales a cada lado, semejantes a rajaduras.

3a. Aberturas branquiales en posición lateral. Espiráculos ausentes.

Carcharhinidae

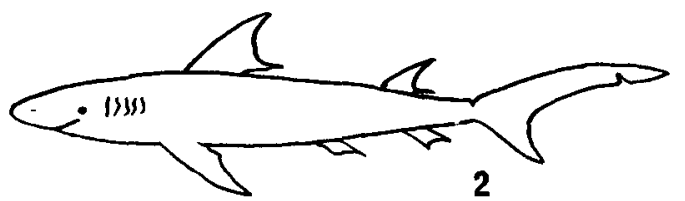

3b. Aberturas branquiales en posición ventral. Espiráculos. Morro prolon. gado en una hoja o sierra con dientes a los lados.

Pristidae

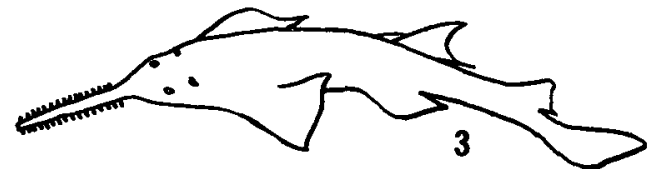


2b. Una sola abertura branquial a cada lado.

4a. Aletas ventrales en posición abdominal no compuestas de una espina y cinco tadios.

5a. Morro alargado. Aleta caudal heterocerca. Escamas romboidales, ganoideas, muy duras. Lepisoste:dae.

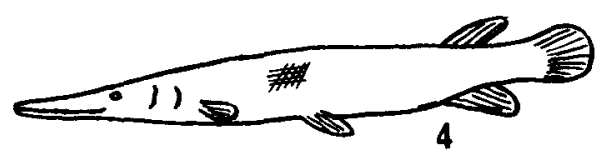

5b. Morro normal. Aleta caudal no heterocerca. Sin escamas $a$ con escamas cicloideas.

6a. Aleta dorsal adiposa presente

7a. Cuerpo sin escamas. Morro y mejilla con barbas. Aleta adiposa más larga que la cabeza. Pimelodidae.

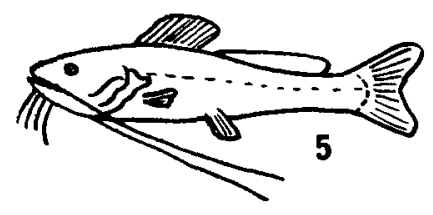

7b. Cuerpo con escamas. Morro y mejilla sin barbas. Aleta adiposa mucho más corta que la cabeza. Characidae

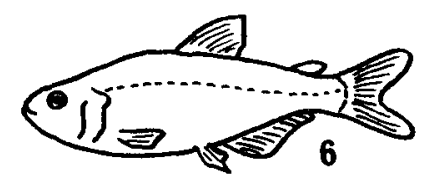

6b. Aleta dorsal adiposa ausente

8a. Ultimo radio de la aleta dorsal prolongado en un filamento. Aleta pectoral insertada mucho más abajo que el eje del cuerpo

9a. Placa gular presente entre las ramas de la mandíbula inferior. Borde ventral no armado con escudo óseo. Megalopidae

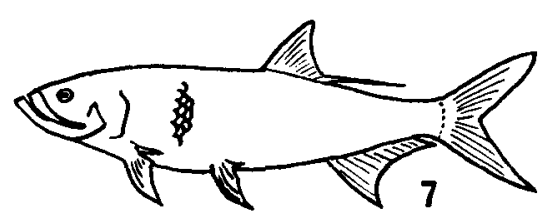


9b. Placa gular ausente. Borde ventral armado con escudos óseos. Clupeidae

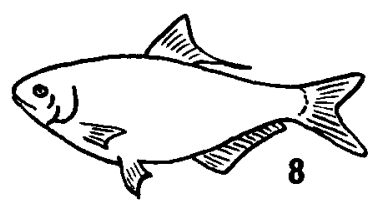

8b. Ultimo radio de la aleta dorsal no prolongado en filamento. Aleta pectoral insertada en, o encima del eje del cuerpo.

10a. Una única aleta dorsal sin espinas. Aleta caudal de borde redondeado. Poeciliidae
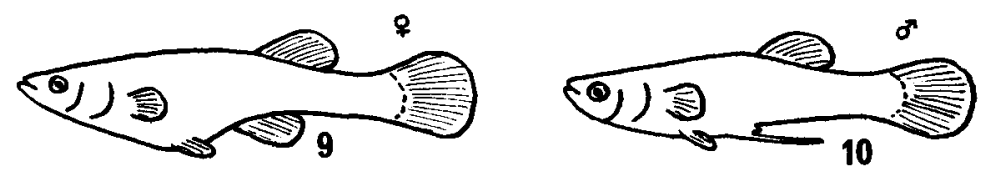

10b. Dos aletas dorsales, la primera con espinas delgadas. Aleta caudal de borde bifurcado. Atherinidae

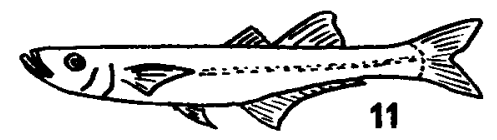

4b. Aletas ventrales en posición toráxica, cada una compuesta de una espina y cinco radios.

11a. Aleta dorsal con más de ocho espinas. Aleta anal con tres o más espinas.

12a. Linea lateral no interrumpida. Doble orificio nasal a cada lado. Tres espinas anales. Pomadasyidae

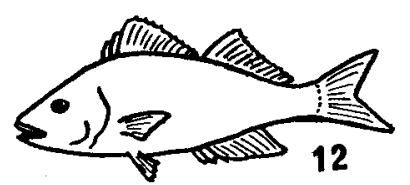

12b. Línea lateral interrumpida a cada lado. Un sólo orificio nasal a cada lado. Más de tres espinas en la aleta anal.

Cichlidac

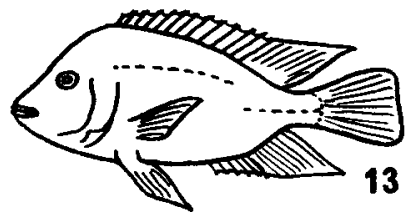


11b. Aleta dorsal con menos de ocho espinas. Sólo una espina en la aleta anal. Eleotridae

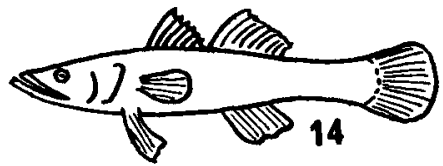

1b. Aberturas branquiales confluentes en una única abertura branquial en posición ventral. Synbranchidae

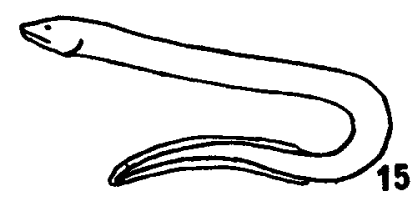

Familia CARCHARHINIDAE

(Fig. 2)

\section{Carcharbinus leucas (Müller \& Henle)}

Carcharias leucas Müller \& Henle, 1841: 42 (descripción original; Antillas)

Eulamia nicaraguensis Gill \& Bransford, 1877: 190, (Descripción original; Lago Nicaragua ); Marden, 1944: 86, (fotografías; Lago Nicaragua).

Carcharias nicaraguensis Lutken, 1879: 65, (descripción; Río San Juan, Nicaragua); Jardan, Evermann \& Clark, 1930: 16, (Lago Nicaragua, Río San Juan).

Carcharbinus nicaraguensis Jordan, 1887: 556, (nombrado); Meek, 1907: 103, (nombrado; Lago Nicaragua y Rio San Juan); Eigenmann, 1910: 377, (nombrado); Smith, 1936: 64, (nombrado como tiburón de agua dulce); Norman \& Fraser, 1937: 36, (aislado en agua dulce); Bigelow \& Schroeder. 1948: 378-82, (referencias, descripción, comparación con C. leucas).

Carcbarbinus (Carcbarbinus) nicaraguensis Jordan \& Evermann, 1896: 39, (descripcibn: Lago Nicaragua y Río San Juan).

Carcbarbinus milberti Meek \& Hildebrand, 1923: 38, (se refiere como sinónimo de $C$. milberti).

No pude obtener ejemplares de esta especie durante la expedición, aunque vi un ejemplar pequeño en Los Cocos, en una laguna poco profunda separada del lago debido a la prolongada sequia. 
Según estudios verificados en los últimos años (Bigelow \& SCHRoeder, 5; ThORSON et al., 48) ya no se reconocen las diferencias, anteriormente citadas por varios ictiólogos, entre Carcharbinus nicaraguensis del lago y $C$. leucas de ias dos costas de las Américas.

\section{Familia PRISTIDAE}

(Fig. 3)

\section{CLAVE PARA LAS ESPECIES DE PRISTIS DEL LAGO NICARAGUA}

1a. La aleta caudal presenta un lóbulo inferior bien definido. De quince a diecinueve pares de dientes en la sierra. El origen de la primera dorsal considerablemente más adelante que el origen de las pélvicas $P$. perotteti

1b. La aleta caudal no presenta un lóbulo inferior bien definido. De veinticinco a treinta y dos pares de dientes rostrales. El origen de la primera dorsal sobre el origen de las pélvicas. P. pectinatus

\section{Pristis perotteti Müller \& Henle}

Pristis perotteti Müller \& Henle, 1841: 108, (descripción; aguas del Senegal); Regan, 1908: 83, (citado; Lago Nicaragua).

Pristis antiquorum Gill \& Bransford, 1877: 190, (sierra; Lago Nicaragua); Meek, 1907: 104, (sierras; Lago Nicaragua).

Pristis microdon Marden, 1944: 184-86, (fotografías en color; Lago Nicaragua).

Esta especie parece ser la más común en el Lago Nicaragua. Entre la colección de once sierras que se examinaron en el Colegio América, en Granada, nueve pertenecían a esta especie, como también todas las sierras de la numerosa colección de Armando Vega, del mismo lugar. La longitud de las sierras es de 200 a $930 \mathrm{~mm}$ y hay de 15 a 18 dientes de cada lado.

El material del Lago Nicaragua, examinado por Bigelow y SCHROEDER, (4), incluye un macho inmaduro de $1.376 \mathrm{~mm}$ de longitud. Según ellos:

Comparado con $P$. pectinatur, está quizás más estrictamente confinado a aguas poco profundas en la inmediata vecindad de la costa y estuarios, lagunas parcialmente cerradas y situaciones similares. Tal vez la zona de la plataforma continental de hasta 15-20 pies de profundidad encierra prácticamente todos los individuos de esta especie a lo largo de su área geográfica a ambos lados del Atlántico. Desde hace mucho se sabe que se encuentra tan a su gusto en agua dulce como en salobre o salada.

Una hembra de $377 \mathrm{~cm}$ de longitud fue capturada y examinada en la Localidad 4. La cola tenía un lóbulo inferior muy definido. La inserción de la aleta pélvica estaba solamente un poco más adelante que el final de la base de la primera dorsal. Los dientes rostrales eran 15 a la derecha y 17 a la izquierda. El color era chocolate claro en el lado dorsal y naranja-amarillo en el ven. tral y en la parte superior de los extremos de las aletas. Esta coloración ana- 
ranjada parece ser una peculiaridad de los peces sierra del Lago. Según los pescadores (confirmado) esta especie cría en el Lago.

$P$. microdon Latham es considerado como un sinónimo de P. perotteli por Bigelow y SCHROFDer (4), quienes indican que:

El pez sierra de la costa del Pacífico de Centro América, que ha sido repetida. mente citado como P. zephyreus Jordan \&: Starks 1895, como P. microdon Latham 1794, y como $P$. perotteti Müller \& Henle 1841, parece distinguible del $P$. perotteti del Atlántico solamente por el hecho de que a veces tiene hasta 23 dientes rostrales en un lado (he. mos visto uno con 20/23) siendo así que la forma Atlántica se supone tener un máximo de 19 a 20.

Según Beebre y The-Van (2), este pez sierra del Pacífico puede tener de 17 a 23 dientes rostrales a cada lado y el $P$. perotteti del Atlántico de 15 a 19 , lo mismo que los peces sierra del Lago. Esto parece reforzar la opinión del autor de que los tiburones y peces sierra del Lago son de origen atlántico y no pacífico.

\section{Pristis pectinatus Latham}

Pristis pectinatus Latham, 1794: 278, (descripción; Océano Atlántico); Bigelow \& Schroeder, 1953: 28, (citado como probablemente viviendo en el Lago Nicaragua).

Según Bigelow y SCHroeder (4), han sido reportadas dos especies de pez sierra en el Lago.

No pude obtener ningún ejemplar de esta especie, pero fueron examinadas dos sierras pertenecientes a la colección del Colegio Centro América. Están anotadas como pertenecientes a peces sierra del Lago; pero no hay ninguna indicación con respecto a la localidad. Una de ellas es de $815 \mathrm{~mm}$ de longitud y $110 \mathrm{~mm}$ de base, a la altura del primer diente proximal, y tiene 27 pares de dientes. La otra es de $312 \mathrm{~mm}$ de largo y 47 de ancho en la base y tiene 29 dientes a cada lado.

Pristis pectinatus no parece ser abundante en el Lago cerca de Granada. Como se ha dicho antes, no hay ni una sierra de esta especie entre las numerosas coleccionadas por Armando Vega cerca de Granada. Podría ocurrir que habitara solamente la parte del Lago cercana al rio San Juan o que llegara sólo de vez en cuando al Lago desde el mar. Esta especie se encuentra con frecuencia en agua dulce corriente arriba de los ríos en diferentes partes del mundo.

\section{Familia LEPISOSTEIDAE}

(Fig. 4)

\section{Lepisosteus tropicus (Gill)}

Atractosteus tropicus Gill, 1863: 172, (descripción original; América Central). 
Lepisosteus tropicus Jordan \& Evermann, 1896: 111, (descripción; costa del Pacífico de Centro América); Meek, 1907: 104, (descripción; ejemplares del Lago Nicaragua); Regan, 1908: 182, (descripción; extensión geográfica); Miller, 1954: 230, (los lagos de Nicaragua límite sur de los lepisósteos).

Esta especie se encuentra en ambos lagos. Vi una serie de más de sesenta ejemplares de gran tamaño en la Localidad No. 4. Habían sido pelados y limpiados y estaban tostándose sobre un fuego al aire libre. La carne así preparada se vende como alimento en el mercado de Granada. Este pez parece ser muy abundante en los lagos, especialmente en las zonas poco profundas y con mucha vegetación cerca del desagüe del Lago de Nicaragua, donde se dice que desova entre la vegetación acuática.

Según Miller (40), el gaspar de los grandes lagos de Nicaragua llegó a ellos desde el oeste, cuando el Lago Nicaragua era una bahía con una salida hacia el Pacífico. Esta afirmación está basada en el conocido habitat de estos peces y en la teoria de Durham (11), que decía que los lagos eran una bahía del Pacífico. Esta última afirmación ha sido recientemente refutada por ZoppisBRACCI y DEL Giudice (49), y con respecto al origen de los gaspares, será necesaria una mayor investigación, especialmente en la vertiente atlántica de $\mathrm{Ni}$ caragua. Pudo también ocurrir que los gaspares llegaran a los lagos desde algún río preexistente en la vertiente del Pacífico y que desaguó en los lagos cuando éstos se formaron. Se coleccionaron tres ejemplares de 493,493 y $555 \mathrm{~mm}$ de longitud, y una cabeza de $270 \mathrm{~mm}$ de largo.

Los dientes son ligeramente curvos, cónicos en la base y lanceolados en los extremos; huesos palatinos con una serie de caninos alargados. Ancho del morro en la base menos que 3.8 de su longitud.

Los tres ejemplares completos dan: longitud total 493-555 (513) $\mathrm{mm}$; longitud de la cabeza 268-272 (270); longitud del morro 161-166 (163); longitud pectoral 274-278 (276); longitud prepélvica 480-485 (482); de la inserción de los pectorales a la inserción de las pélvicas 232-245 (238); diámetro orbital 25.2-28.4 (27); ancho interorbital 59-67 (64); escamas laterales 51-52 (51.13); radios dorsales 6-7; radios anales 6-7; rayos caudales 12 .

\section{Familia PIMELODIDAE}

(Fig. 5)

En el Lago parece que existen tres especies muy próximas y aparentemente el único criterio para distinguirlas es la longitud de las barbas. La profundidad del cuerpo, usada por MEeK (34) como uno de los caracteres para separar Rhamdia managuensis de las otras dos especies no es útil puesto que los ejemplares jóvenes de $R$. nicaraguensis y de $R$. barbata tienen cuerpos tan delgados como los de $R$, managuensis. El número de radios anales, carácter usado por GuNTHER (21) y JoRdan y EVERMANN (28) para separar $R$. managuensis de $R$. nicaraguensis no parece servir tampoco. Según estos autores $R$. managuensis tiene de 14 a 15 rayos anales; mientras que MEEK (34) dice que tiene 12. Las 
otras especies, $R$. nicaraguensis y $R$. barbata tienen de 11 a 13 como aparece en nuestros ejemplares.

Sería necesaria una colección mucho más representativa para poder hacer una mejor interpretación de estas especies. Por ahora, la longitud de las barbas maxilares se usará como el único criterio (provisional).

\section{CLAVE PARA EL GENERO RHAMDIA}

1a. Barbas maxilares no llegan a la mitad de la base de la aleta dorsal. $R$. managuensis

1b. Barbas maxiliares llegan cerca, $y$ aun sobrepasan el origen de la aleta adiposa dorsal. R. nicaraguensis

1c. Barbas maxilares pasan de la mitad de la aleta adiposa dorsal. R. barbata

\section{Rhamdia managuensis (Günther)}

Pimelodus managuensis Günther, 1866: 603, (descripción original; Lago Managua).

Rhamdia managuensis, Jordan \& Evermann, 1896: 153, (descripción; Lago Managua); Regan, 1908: 133, (descripción: Lago Managua); Meek, 1907: 105, (descripción; Lago Managua).

\section{Rbamdia nicaraguensis (Günther)}

Pimelodus nicaraguensis Günther, 1864; 125, (descripción original; lagos de Nicaragua).

Rbamdia nicaragkensis, Gill \& Bransford, 1877: 190, (enumerado); Jordan \& Evermann, 1896: 152, (descripción; Lago Nicaragua); Meek, 1907: 106, (descripción; Lago Managua); Regan, 1908: 130, (descripción; Lago Nicaragua).

Esta especie parece ser la más abundante y la que con más frecuencia se encuentra en el mercado de Granada. La siguiente descripción está basada en once ejemplares: ocho de 142 a $253 \mathrm{~mm}$ (L.1); uno de $98 \mathrm{~mm}$ (L. 18); uno de $90 \mathrm{~mm}$ (L. 35); uno de $96 \mathrm{~mm}$ (L. 12).

Color verdoso; línea lateral poco visible, una banda blanca a través de la aleta dorsal, muy borrosa en los ejemplares jóvenes; en los grandes no aparecía esa banda; aleta caudal profundamente bifurcada. El extremo de la aleta anal plegada no llega al nivel del final de la aleta dorsal adiposa.

D. I,9; A. 11-12; filamentos branquiales 3-4, 8-11; longitud predorsal 334-364 (348); longitud preanal 654-735 (680); longitud prepélvica 444-517 (473); longitud de la base de la aleta dorsal 109-142 (126); longitud de la base de la aleta anal 93-143 (123); longitud de la dorsal 174-226 (208); longitud de la aleta anal 153-204 (184); longitud de la aleta adiposa 355-423 (386); distancia desde la punta del morro hasta el origen de la aleta adiposa 490-553 (521); longitud de lóbulo caudal superior 162-225 (194); longitud del lóbulo caudal inferior 156-204 (186); longitud de la espina pectoral 89-124 (107); longitud de la cabeza 230-262 (247); ancho de la cabeza 174-235 (191); longitud del morro 91-101 (98); ancho del morro 96-131 (110); distancia inter- 
orbital 52-89 (69); diámetro orbital $33-52$ (44); profundidad del cuerpo 156206 (180); parte más estrecha del pedúnculo caudal 82-127 (94); longitud de las barbas maxilares 435-675 (520).

\section{Rhamdia barbata Meek}

Rbamdia baibata Meek, 1907; 106, (descripción original; Lagos Managua y Nicaragua); Regan, 1908: 191, (descripción; Lago Managua).

La descripción que sigue está basada en tres ejemplares: dos de 112 y $152 \mathrm{~mm}$ (L. 12); uno de $120 \mathrm{~mm}$. (L. 36).

Color verdoso, más oscuro que $R$. micaraguensis; línea lateral oscura y bien marcada en dos ejemplares, muy borrosa en el tercero; una banda de color bien visible en la aleta dorsal. Aleta caudal profundamente bifurcada; lóbulo caudal inferior un poco más largo que el superior.

D. I,6; A. 11-12; filamentos branquiales 3-4, 7-8; longitud predorsal 321-350 (333); longitud preanal 660-683 (669); longitud prepélvica 455483 (470); longitud de la base de la aleta dorsal 135-145 (140); longitud de la base de la aleta anal 125-135 (131); longitud de la aleta dorsal 203-228 (216); longitud de la aleta anal 184-192 (188); longitud de la aleta adiposa 400-441 (423); distancia desde la punta del morro hasta el origen de la aleta adiposa 482-510 (499); longitud del lóbulo caudal inferior 217-250 (230); longitud de la espina pectoral 93-113 (104); longitud de la cabeza 243-251 (246); ancho de la cabeza 183-191 (187); longitud del morro 98-107 (101); ancho del morro 116-118 (117); distancia interorbital 72-82 (76); diámetro orbital 42-47 (44); profundidad del cuerpo 187-194 (190); parte más estrecha del pedúnculo caudal 99-107 (103); longitud de las barbas maxilares 822-966 (896).

\section{Familia CHARACIDAE}

(Fig. 6)

\section{CLAVE PARA LOS GENEROS}

(modificada de Meek \& Hildebrand, 37)

1a. Dientes de la mandíbula superior en una serie

2a. Dientes de forma canina, los delanteros salientes hacia adelante Bramocharax

2b. Dientes con la corona aplanada y ensanchada en su parte superior, con varios picos, al mismo nivel. Rboadsia

1b. Dientes de la mandibula superior en un número de series de dos a cuatro.

32. Dientes de la mandíbula superior en dos series

4a. Línea lateral incompleta Hyphessobrycen

4b. Línea lateral completa 
5a. Maxilares con ninguno o pocos dientes

6a. Segundo hueso suborbital estrecho, dejando una área triangular desnuda debajo de la sutura entre el primero y segundo suborbitales Astyanax

6b. Segundo suborbital ensanchado, no dejando una área triangular desnuda debajo de la sutura entre el primero y segundo sub. orbitales. Bryconamericus

5b. Maxilares, en los adultos, con 5 a 25 dientes Hemibrycon

3b. Dientes en la mandibula superior en 3 ó 4 series

7a. Mucho más de 40 radios en la aleta anal. Cuerpo muy comprimido lateral. mente. Rosboider

7b. Menos de 40 radios en la aleta anal. Cuerpo fusiforme. Brycon

\section{Bramocharax bransfordii Gill \& Bransford}

Bramocharax bransfordii Gill \& Bransford, 1877: 189, (descripción original; Lago Nicaragua); Jordan \& Evermann, 1896: 339, (descripción según Gill); Eigenmann \& Ogle, 1907: 32, (descripción, fotografia); Meek, 1907: 110, (cnumerado).

Bramocbarax elongatus Meek 1907: 110 (descripción original; Lago Managua); Regan, 1908: 174, (descripción).

La siguiente discusión y descripción está basada en veinte ejemplares: cuatro de 92 a $118 \mathrm{~mm}$ (L. 2); trece de 69 a $133 \mathrm{~mm}$ (L. 13); tres de 81 a 85 $\mathrm{mm}$ (L. 24).

MeEk (34) describió a $B$. elongatus como una nueva especie diferente a $B$. bransfordii en la relativa profundidad del cuerpo. En los ejemplares del presente estudio la profundidad relativa del cuerpo decrece con la edad. Nuestra serie incluye ejemplares de 69 a $133 \mathrm{~mm}$ (los de Meek son de 11 a $150 \mathrm{~mm}$ ). La profundidad relativa del cuerpo en nuestros ejemplares es de 3 a 3.7 , es decir, un término medio entre las profundidades dadas para $B$. bransfordii (2.7) y $B$. elongatus (3.4-3.9). Esto pudiera indicar que $B$. elongatus es el adulto de $B$. bransfordii. Sin embargo, será necesario conocer la longitud del ejemplar tipo descrito por Gill (17) para poder asegurar que se trata de un ejemplar joven. No hay ninguna indicación de la longitud en la descripción original.

Una sola serie de dientes en cada mandíbula; los dos dientes delanteros en cada mandíbula alargados y de forma canina; los de la mandíbula superior sobresaliendo sobre el borde de la mandíbula inferior, formando dos huecos en el labio inferior; los otros dientes con picos en la base, especialmente en la maxilar, que presenta una fila a lo largo de casi toda su longitud. La línea lateral completa y curvada. La aleta caudal bifurcada. El color verdoso más oscuro en la parte superior presenta una mancha humeral; banda oscura lateral que termina en la cola en una mancha. El morro se hace alargado y puntiagudo con la edad. 
D. 11; A. 27-32; Pct. 13-16; escamas a lo largo de la línea lateral, 35-38; filamentos branquiales 7-10, 14-17.

Longitud estándard 69-133 mm (99); longitud predorsal $492-536$ (516); longitud preanal 630-693 (655); longitud prepélvica 478-528 (496); profundidad del cuerpo 273-336 (305); parte más estrecha del pedúnculo caudal 96109 (102); longitud de la cabeza 286-334 (312); longitud del morro 73-98 (87); diámetro orbital 60-94 (78); distancia interorbital 65-84 (74); longitud del maxilar 128-164 (145), altura de la aleta dorsal 222-250 (235); altura de la aleta anal 129-174 (142); longitud de la aleta pectoral 165-222 (197); longitud de la aleta pelviana 135-174 (150); longitud del lóbulo caudal superior 238-288 (263); distancia desde la punta del morro hasta el origen de la aleta adiposa $860-895(870)$.

Material: 2 ej. (L. 14); 1 ej. (L. 12); 4 ej. (L. 28); 14 ej. (L. 13); 4 ej. (L. 2); 1 (L. 1); 3 ej. (L. 24).

\section{Rhoadsia eigenmanni Meek}

Sólo se coleccionó tres ejemplares: uno de $43.5 \mathrm{~mm}$ (L. 36); dos de 38 y $40.5 \mathrm{~mm}$ (L. 12).

Color amarillento; algunos melanóforos, sobre todo en la base de la aleta caudal; mancha humeral situada al final de la línea lateral incompleta; mancha caudal de forma romboidal, bien marcada, se extiende por los radios centrales.

Suborbital estrecho; morro redondeado; extremo del maxilar llega a la vertical que pasa por la parte delantera de la pupila.

Dientes comprimidos, con nueve vértices redondeados, el vértice central algo más largo, colocados en una sola fila en cada mandíbula; extremo posterior del maxilar redondeado con dos dientes en el ángulo superior; de cinco a seis dientes a cada lado de la mandíbula, que se superponen lateralmente y sobresalen algo.

Origen de la aleta anal debajo de la mitad de la base de la aleta dorsal; aleta pectoral sobrepasa el origen de la pélvica que a su vez sobrepasa el origen de la anal; aleta caudal bifurcada, subigual, el lóbulo superior es más corto.

D. 11; A. 33-35; Pct. 13; escamas a lo largo de la línea lateral 35-38; filamentos branquiales $6: 10$.

Longitud estándard 43.5.40.5 mm (40.6); longitud predorsal 515-543 (529); longitud preanal 566-589 (578); longitud prepélvica 420-450 (431); profundidad del cuerpo 368-376 (371).

Parte más estrecha del pedúnculo caudal 108-115 (112); longitud de la cabeza 244-251 (248); longitud del morro 48-64 (57); diámetro de la órbita 89-100 (95); distancia interorbital 85-89 (87); longitud del maxilar 87-94 (89); altura de la aleta dorsal 296-320 (305); altura de la aleta anal 173-185 (181); longitud de la aleta pectoral 188-198 (194); longitud de la aleta pélvica 161-175 (169); distancia desde la punta del morro hasta el origen de la aleta 
adiposa 860-864 (862).

Estos ejemplares que primeramente fueron considerados como posiblemente pertenecientes al género Pseudocheirodon, aunque difieren de $P$. affinis por tener un número mayor de escamas (35-38 contra 31-33), mayor número de radios anales (30-32 contra 21-24) y la posición de la aleta dorsal y el ancho del segundo suborbital como se ha indicado arriba, han sido finalmente identificados por el Profesor L. R. Rivas como pertenecientes a la especie $R$. eigenmanni.

\section{? Hyphessabrycon tortuguerae Böhlke}

Hyphessobyycon tortuguerae Böhlke, 1958, (descripción original; Río Tortuguero, Costa Rica).

La descripción que sigue está basada en quince ejemplares (L. 24). Son mayores que los tipos descritos por BöHLKE (8) (46.5-55 mm contra 24.2-39 mm) pero concuerdan con su descripción.

Cuerpo cubierto de melanóforos dando al pez una apariencia como de estar embarrado. La línea lateral es muy borrosa y no hay mancha caudal. En algunos de los ejemplares aparece una línea oscura a través de las aletas anal y caudal.

Dientes con tres vértices; colocados en dos filas en la mandíbula superior, la fila más externa formada por dos dientes y la más interna por seis o siete en cada lado. La mandíbula inferior avanza sobre la superior y presenta a cada lado cinco dientes grandes y seis pequeños en una soia fila. En el maxilar hay de siete a once dientes ocupando más de dos tercios de su longitud.

D. 11-12; A. 26-31; Pct. 12-14; escamas en la línea lateral 34-37; filamentos branquiales $8-10,16-18$.

Longitud estándard 46.5-55 mm (50); longitud predorsal 490-538 (516); longitud preanal 580-624 (608); longitud prepélvica 444-470 (456); profundidad del cuerpo 342-400 (370); parte más estrecha del pedúnculo caudal 102-120 (110); longitud de la cabeza 270-288 (276); longitud del morro 53-63 (58); diámetro de la órbita 95-107 (99); distancia interorbital 69-82 (77); longitud del maxilar 107-122 (114); altura de la aleta dorsal 250-291 (270); altura de la alcta anal 161-204 (181); longitud de la aleta pectoral 173-210 (199); longitud de la aleta pelviana 177-204 (192); distancia desde el extremo del morro hasta el comienzo de la aleta adiposa 820-865 (842).

Esta especie no había sido previamente enumerada entre los peces de $\mathrm{Ni}$ caragua. Durante la estación de las lluvias, debido a inundaciones costeras, es posible que haya un fácil intercambio de la población de peces entre el río Tortuguero y el sistema fluvial del río San Juan.

\section{CIAVE PARA LAS ESPECIES DE ASTYANAX}

1a. Cuerpo delgado; profundidad incluida más de 3 veces en la longitud estándard. La banda lateral muy oscura y la mancha humeral borrosa. La boca con una marcada inclinacion hacia arriba.

Astyanax sp. 
1b. Cuerpo profundo; profundidad menos de 3 veces en la longitud estándard. La banda lateral más clara y la mancha humeral más marcada. La boca más pequeña y sin tanta inclinación hacia arriba. Astyanax fasciatus

\section{Astyanax sp.}

La discusión y descripción que sigue se basa en diecinueve ejemplares: trece de 38 a $49 \mathrm{~mm}$ (L. 1); cuatro de 41 a $47 \mathrm{~mm}$ (L. 12); dos de $47 \mathrm{~mm}$ (L. 36).

Esta especie se encuentra juntamente con A. fasciatus, pero se le puede distinguir fácilmente por la apariencia externa del cuerpo. Como se hace notar en la clave, hay una diferencia muy marcada en la forma de la boca, además de tener un cuerpo más fino y la banda lateral mucho más oscura. No se encontró diferencias apreciables en cuanto a las demás proporciones del cuerpo y número de escamas y radios.

Los dientes son semejantes a los de fasciatus; dos serics con tres vértices en el maxilar, el vértice superior algo más largo. En el ángulo superior del maxilar uno o dos dientes comprimidos con muchos véttices. En la mandibula una sola serie de dientes que presentan dos o cuatro pequeñas cúspides basales.

D. 10-11; A. 26-30; Pct. 12-14; escamas en la línea lateral 34-36; filamentos branquiales 10-12, 16-20.

Longitud predorsal 480-522. (501); longitud preanal 610-682 (644); longritud prepélvica 456-503 (474); profundidad del cuerpo 282-340 (313); parte más estrccha del pedúnculo caudal 91-107 (101); longitud de la cabeza 261-289 (272); longitud del morro 49-63 (54); diámetro de la órbita 83-109 (92); distancia interorbital 65-77 (70); longitud del maxilar 96-113 (104); altura de la aleta dorsal 224-275 (254); altura de la aleta anal 146-186 (163); longitud de la aleta pectoral 185-239 (198); longitud de la pelviana 135-174 (158); distancia del morro al comienzo de la aleta adiposa 830-870 (851).

Según Rrvas (comunicación personal) tal vez se trate de $A$, nicaraguensis.

\section{Astyanax: fusciatus (Cuvier)}

Chalceus fasciatus Cuvier, 1819: 352, (descripción original; Brasil).

Tetragonopterus aeneus Günther, 1860: 319, (descripción original; Oaxaca, México); Regan, 1908: 170, (referencia, sinonimia, descripción; Lagos Managua y Nicaragua).

Astyanax aenews Meek, 1907: 108, (descripción; Lagos Managua y Nicaragua); Fowler, 1923: 26, (descripción; Nicaragua).

Astyanax rutilus nicaraguensis Eigenmann \&: Ogle, 1907: 23, (descripción; Nicaragua).

Astyanax nasutus Meek, 1907: 108, (descripción; Lagos Managua y Nicaragua). 
Todos los ejemplares de la colección parecen pertenecer a la misma especie. Se han descrito tres especies de Astyanax de Nicaragua: $A$. nasutus Meek, A. deneus Meek (34) y A. rutilus nicaraguensis Eigenmann \& Ogle. Los tratados acerca del género Astyanax son muy confusos y se hace necesaria una revisión del género. Nuestros ejemplares parecen pertenecer a la especie descrita por Meek como $A$. deneus. La única diferencia entre esta especie y $A$. nasutus parece ser la longitud del morro y del maxilar. Según MeEK (34):

1a. Morro alargado, su longitud incluida de 3.3 a 3.5 veces en la longitud de la cabe$z a, y$ longitud del maxilar incluida de 2 a 2.2 veces. ? A. nasutus

1b. Morro corto, su longitud incluida de 3.7 a 4.2 veces en la longitud de la cabeza y Inngitud del maxilar incluida de 2.3 a 2.6 veces. A. aeneus

Entre los ejemplares de nuestra colección se puede establecer una clara gradación para la longitud del morro que va de 3.4 a 4.9 y para la longitud del maxilar de 2.2 a más de 3 . Por esta razón por ahora consideramos a $A$. nasutus como la misma especie que $A$. aeneus. Según Rivas y Myers (comunicación personal) nuestros ejemplares de $A$. nasutus pertenecen en realidad a un nuevo género Detuterodon nasutus (Meek). REGAN (44) considera a $A$. aeneus como sinónimo de $A$. fasciatuss; por otra parte $A$. fasciatus se puede distinguir de la otra especie por no tener mancha caudal. EIGENMANN (12) considera los ejemplares citados por MEeK \& Hildebrand (37) como A. fasciatus, debido a que presentan mancha caudal, aunque muy borrosa.

Dado que las claves publicadas hasta ahora son tan confusas, el autor prefiere seguir a REGAN (44) hasta que no se haga una revisión, y considera los ejemplares como $A$. fasciatus.

La descripción que sigue está basada en cuarenta y cinco ejemplares: veinticinco de 26 a $82 \mathrm{~mm}$ (L. 17); veinte de 43 a $100 \mathrm{~mm}$ (L. 13).

Color plateado, verdoso oscuro en el dorso; banda lateral bien marcada desde la parte superior del opérculo hasta la cola, formando en la base de esta última una mancha alargada que se extiende por los radios del medio de la cola.

Dos filas de dientes en la mandibula superior, y una fila en la mandíbu. la inferior, todos con cinco vértices, siendo el del medio algo más largo; uno a cuatro dientes aplanados en la parte superior del maxilar; boca pequeña; el maxilar se extiende hasta la vertical trazada por el primer tercio de la órbita.

Se examinaron dos series de los Lagos Managua y Nicaragua, respectivamente. Se pueden observar algunas diferencias en cuanto a las proporciones del cuerpo entre estas dos series; esto se puede deber, en parte, al tamaño más pequeño de los ejemplares del Lago Nicaragua.

Las proporcions y números para los ejemplares del lago Nicaragua son los siguientes: D. 11 ; A. 26-32; Pct. 12-14; escamas a lo largo de la línea lateral 36-40; filamentos branquiales 9-11, 13-16.

Longitud estándard 26-82 mm (48); longitud predorsal 497-600 (520); longitud preanal 614-729 (644); longitud prepélvica 450-538 (480); profundidad del cuerpo 322-405 (351); parte más estrecha del pedúnculo caudal 94-128 
(112); longitud de la cabeza 252-332 (285); longitud del morro 58-82 (68); diámetro orbital 74-125 (100); distancia interorbital 81-96 (88); longitud del maxilar 99-126 (112); alutura de la aleta dorsal 233-308 (274); altura de la aleta anal 134-180 (165); longitud de la aleta pectoral 195-250 (217); longitud de la aleta pelviana 143-190 (162); distancia de la punta del morro al origen de la aleta adiposa 831-900 (859).

Las proporciones y números para la serie del Lago Managua son como sigue: D. 11; A. 27-30; Pct. 12-14; escamas a lo largo de la linea lateral 34-39; filamentos branquiales 8-11, 12-19.

Longitud estándard 43-100 mm (64); longitud predorsal 490-540 (509); longitud preanal 607-674 (643); longitud prepélvica $443-490$ (465); profundidad del cuerpo 323-400 (351); parte más estrecha del pedúnculo caudal 99125 (113); longitud de la cabeza 243-282 (264); longitud del morro 53-68 (60); diámetro de la órbita 71-100 (91); distancia interorbital 67-90 (78); longitud del maxilar 88-115 (103); altura de la aleta dorsal 235-266 (254); altura de la aleta anal 137-186 (160); longitud de la aleta pectoral 193-226 (211); longitud de la aleta pelviana 151-188 (166); distancia desde la punta del morro hasta el origen de la aleta adiposa 826-869 (850).

Material: 2 ej. (L. 1); 21 ej. (L. 2); 13 ej. (L. 3); 31 ej. (L. 5); 4 ej. (L. 12); 33 ej. (L. 13); 1 ej. (L. 14); 24 ej. (L. 15); 46 ej. (L. 17); 5 ej. (L. 15); 46 ej. (L. 17); 5 ej. (L. 18); 8 ej. (L. 24); 36 ej. (L. 28); 1 ej. (L. 29); 1 ej. (L. 30); 6 ej. (L. 31); 45 ej. (L. 35); 7 ej. (L. 36).

\section{Bryconamericus ricae Eigenmann}

Brjconamericus peruanus ricae Eigenmman, 1908: 106, (descripción original; cuenca del Río Reventazón, Costa Rica).

Esta especie se describe por primera vez entre los peces de los lagos de Nicaragua. La descripción que sigue está basada en siete ejemplares: cuatro de 54 a $78 \mathrm{~mm}$ (L. 35); tres de 58 a $66 \mathrm{~mm}$ (L. 36).

Color oscuro, manchas formadas por pequeños puntos en todo el cuerpo; mancha humeral muy borrosa; banda lateral ancha y abriéndose en una mancha caudal no muy marcada en los ejemplares grandes.

Dientes como los de Astyanax pero algo más fuertes. Según Hildebrand (26):

Esta difiere de las otras especies hasta ahora encontradas en Panamá, por la posición más anterior de la aleta dorsal, el origen de la cual se apunta que está en una distancia igual al diámetro del ojo más cerca del morro que de la base de la cola. $\mathrm{Pa}_{\text {- }}$ rece probable por lo que se ha publicado que $B$. ricae puede tener más escamas y más ra. dios anales que las otras formas de Panamá, las cantidades que se indican, son: escamas 39-40; radios anales $28-31$.

Nuestros ejemplares tienen el origen de la aleta dorsal algo más cerca de la punta del morro que de la base de la aleta caudal, pero sólo dos por una distancia tan grande como el diámetro orbital. 
D. 10-11; A. 30-32; Pct. 12-13; escamas línea lateral 37-40; filamentos branquiales $6-9,10-15$.

Longitud estándard 54-78 mm (65); longitud predorsal 480-521 (504); longitud preanal 590-635 (613); longitud prepélvica 440-476 (460); profundidad del cuerpo 332-371 (358); parte más estrecha del pedúnculo caudal 110136 (122); longitud de la cabeza 257-276 (265); longitud del morro 56-68 (63); diámetro de la órbita 85-95 (91); distancia interorbital 79-91 (85); longitud del maxilar 106-113 (109); altura de la aleta dorsal 254-276 (259); altura de la aleta anal 143-174 (164); longitud de la aleta pectoral 195-223 (214); longitud de la aleta pelviana 142-166 (152); distancia de la punta del morro al origen de la aleta adiposa 830-860 (843).

\section{?Hemibrycon sp.}

La siguiente discusión y descripción se basa en veinticinco ejemplares de 52 a $75 \mathrm{~mm}$ (L. 2). Incluyo los ejemplares de la colección en este género por ser el género en el que mejor encajan. Sin embargo difieren de la descripción general (MEEK \& HILDEBRAND, 37) por tener suborbitales estrechos. Los ejemplares se diferencian claramente de Astyanax por tener numerosos dientes en el maxilar y porque los suborbitales no dejan un espacio triangular desnudo entre el primero $y$ el segundo.

Parte superior del cuerpo oscura; banda lateral oscura, ensanchándose en una mancha romboidal bien marcada en la base de la cola, y extendiéndose por los radios medios; mancha humeral bien marcada, separada del extremo proximal de la banda lateral por un espacio claro.

Boca grande; labio inferior saliente formando un pliegue sobre el labio superior; párpados adiposos bien desarrollados. Dientes puntiagudos con bases provistas de varias cúspides; dos filas de dientes en el premaxilar; fila externa formada a cada lado por dos o tres dientes muy pequeños colocados muy irregularmente; fila interior formada por cuatro grandes dientes a cada lado; siete a once dientes, comprimidos, de varios vértices, a cada lado en el maxilar; cuatro a seis dientes pequeños a cada lado de la mandíbula.

D. 11; A. 27-32; Pct. 12-15; escamas línea lateral 35-40; filamentos branquiales 7-10, 13-16.

Longitud estándard $52-76 \mathrm{~mm}$ (68); distancia predorsal $472-536$ (504); distancia preanal 594-675 (635); distancia prepélvica 432-500 (472); profundidad del cuerpo 286-352 (324); parte más delgada del pedúnculo caudal 97116 (107); longitud de la cabeza 269-314 (286); longitud del morro 55-83 (70); diámetro de la órbita $75-97$ (87); distancia interorbital 70-83 (76); longitud del maxilar 97-128 (118); altura de la aleta dorsal 215-264 (244); altura de la aleta anal 128-174 (151); longitud de la aleta pectoral 187-223 (204); longitud de la aleta pelviana 135-184 (153); distancia de la punta del morro al origen de la aleta adiposa 840-880 (856). 


\section{Roeboides guatemalensis Günther}

Anacyrtus (Roeboides) guatemalensis Günther, 1864: 347, (descripción original; Río Chagres, Panamá; Huamuchal, Guatemala).

Roeboides guatemalensis Jordan \& Evermann, 1896: 338, (descripción, Rio Chagres, Pa. namá; Guatemala); Meek, 1907: 111, (descripción; Lagos Nicaragua y Managua); Regan, 1908: 174, (descripción).

Este parece ser uno de los peces más comunes en los lagos y ríos de $\mathrm{Ni}$ caragua. El siguiente estudio se basa en veinticinco ejemplares de 20 a 106 mm (L. 5).

El cuerpo es muy comprimido; cn los ejemplates más grandes la región dorsal es elevada, tomando un perfil anterior sigmoideo. Cuando vivos son plateados, de color verdoso en su parte dorsal; una serie de finos puntos negros se extiende a lo largo de la base de las aletas impares y por la cabeza; banda lateral plateada, que termina en la base de la cola en un triángulo formado pot numerosos puntitos negros. Aleta caudal desnuda, el lóbulo inferior más largo. Tres series más o menos irregulares de dientes en el pre-maxilar, los de la fila externa cónicos y dirigidos hacia afuera.

Longitud estándard 20-106 mm (75); distancia predorsal 500-530 (516); distancia preanal 450-488 (468); distancia prepélvica 320-380 (347); profundidad del cuerpo 300-371 (340); parte más estrecha del pedúnculo caudal $71-90$ (84); longitud de la cabeza 250-302 (272); longitud del morro 58.90 (73); diámetro de la órbita 71-101 (79); longitud del maxilar 108-131 (121); altura de la aleta dorsal 226-304 (260); altura de la aleta anal 127-185 (149); longitud de la aleta pectoral 172-238 (198); longitud de la aleta pelviana 151-214 (188); longitud del lóbulo caudal superior $217-320$ (258); distancia de la punta del morro al origen de la aleta adiposa 840-890 (862).

Además de Roeboides guatemalensis se ha descrito otras tres especies de Roeboides de Centro América; sin embargo, sus descripciones originales hacen pensar que se trata de variantes geográficas de la misma especie.

Roeboides bouchellei Fowler, del Río Pis-Pis en Nicaragua, es, según el autor (16), "emparentado con $R$. guatemalensis Günther de Centro América, del cual difiere por tener escamas más grandes 55 a $74 \ldots$ por la presencia de una mancha negra cerca y sobre la línea lateral aproximadamente a mitad del camino entre el morro y la aleta dorsal." El número de escamas en nuestros ejemplares va de 73 a 79 y presentan una mancha lateral, como la descrita, aunque algo difusa.

Nuestros ejemplares concuerdan con $R$. occidentalis Meek \& Hildebrand en cuanto al número de escamas; pero en la clave de los mismos autores (37) se identifican como $R$. guatemalensis.

La comparación de nuestros ejemplares con la descripción de $R$. salvadoris Hildebrand (25) está basada en el estudio de esta especie y sus tiferencias con $R$. occidentalis y $R$, guatemalensis que hace el citado autor. Los ejemplares coinciden con $R$. guatemalensis en color, tamaño de los ojos y longitud de las ale- 
tas, todo lo cual parece ser el principal criterio para distinguir las especies arriba citadas.

Material: 56 ej. (L. 5); 10 ej. (L. 4); 1 ej. (L. 3); 11 ej. (L. 12); 1 ej. (L. 17); 13 ej. (L. 18); 1 ej. (L. 15); 24 ej. (L. 28); 3 ej. (L. 29); 5 ej. (L. 30); 3 ej. (L. 35).

\section{Brycon guatemalensis Regan}

Brycon dentex Günther, 1860: 240, (descripción original; Río Esmeralda, Ecuador); Jordan \& Evermann, 1896: 337, (descripción; Esmeraldas, Ecuador); Meek, 1907: 109. (descripción; Lagos Nicaragua y Managua).

Chalcinopsis dentex Günther, 1864: 337; 1868: 478, (descripción); Gill y Bransford, 1877: 188, (descripción; Lago Nicaragua).

Brycon guatemalensis Regan, 1908: 168, (descripción; Guatemala); Fowiler, 1923: 26, (descripción; costa atlántica de Nicaragua).

Como puede verse en las referencias citadas más arriba, los ejemplares de Brycon de los lagos de Nicaragua fueron considerados por RrGan (44) como pertenecientes a $B$. guatemalensis.

La descripción y estudio que sigue se basa en dieciocho ejemplares: tres de $270-378 \mathrm{~mm}$ (L. 1); uno de $104 \mathrm{~mm}$ (L. 5); dos de 75 y $78 \mathrm{~mm}$ (L. 18); dos de 114 y $158 \mathrm{~mm}$ (L. 28); dos de 56 y $91 \mathrm{~mm}$ (L. 29); tres de 84 a 97 mm (L. 31); uno de $85 \mathrm{~mm}$ (L. 35); cuatro de 65 a $306 \mathrm{~mm}$ (L. 36).

Según RFGan, (44):

Los tipos de $B$. dentex, del oeste de Ecuador, tienen 18 filamentos branquiales en la rama inferior del arco anterior, de 47 a 50 escamas en una fila a lo largo del pez, la mandíbula inferior más corta, los ojos más grandes y la región interorbital más estrecha que en B. guatemalensir, y el cuerpo uniformemente plateado.

Una cuidadosa comparación de nuestros ejemplares con la descripción original de $B$. dentex nos lleva a la conclusión de que los nuestros tienen el cuerpo más fino, la cabeza más larga y la región interorbital más ancha. Sin embargo uno de los ejemplares de $450 \mathrm{~mm}$ de largo tiene la misma altura de cuerpo, tres ejemplares de 200, 230 y $400 \mathrm{~mm}$ tienen el mismo largo relativo de cabeza y seis ejemplares todos de menos de $420 \mathrm{~mm}$ de largo, tienen el mismo ancho interorbital que el pez "tipo" de Günther.

En lo que respecta a las otras diferencias señaladas por RFGan (44) Brycon de Ecuador oeste tiene los ojos más grandes. El da como medida de los ojos para $B$. guatemalensis $4-1 / 3$ a $5-1 / 2$ veces en la longitud de la cabeza. El tamaño de los ojos de nuestros ejemplares va de $2-4 / 5$ a $5-1 / 5$.

El número de escamas a lo largo de la línea lateral es de 47 a 50 en el $B$. dentex de Günther $y$ de 49 a 57 en los ejemplares coleccionados. El número de filamentos branquiales en la rama inferior del primer arco es 18 en $B$. dentex Günther y de 14 a 17 en los ejemplares de nuestra colección. 
Con respecto al color cabe notar que algunos de nuestros ejemplares son uniformemente plateados y otros mucho más oscuros en la parte superior del cuerpo. Por lo tanto parece que B. guatemalensis tal vez no sea más que un variante geográfico (subespecie?) de $B$. dentex. Los ejemplares recolectados serán considerados como B. guatemalensis hasta que no se pueda hacer una colección más completa y una más directa comparación entre los ejemplares de Nicaragua y Ecuador.

Color plateado, generalmente más oscuro en la parte superior del cuerpo, una mancha más o menos clara en el pedúnculo caudal. Mandíbula inferior más corta que la superior; cuatro filas de dientes de tres vértices en el premaxilar, la fila más interna encaja con la única fila de dientes que presenta la mandíbula inferior.

D. 11; A. 34-39; Pct. 12-14; escamas línea lateral 49-57; filamentos branquiales en la rama inferior del primer arco 14-17.

Longitud estándard $56.378 \mathrm{~mm}$ (152); distancia predorsal $516-560$ (534); distancia preanal 590-675 (621); longitud prepélvica 436-480 (457); profundidad del cuerpo 275-324 (295); parte más estrecha del pedúnculo caudal 82-100 (91); longitud de la cabeza 244-284 (268); largo del morro 66-86 (74); diámetro orbital 52-98 (79); distancia interorbital 80-119 (90); longitud maxilar 111-135 (121); altura de la aleta dorsal 177-227 (204); altura de la aleta anal 131-181 (148); longitud de la base de la aleta dorsal 104-129 (116); longitud de la base de la aieta anal 276-312 (297); longitud de la aleta pectoral 193-234 (206); longitud de la alcta pelviana 1.31-161 (148); longitud del lóbulo caudal superior 225-298 (265); longitud del lóbulo caudal inferior 274316 (295); distancia desde la punta del morro hasta el origen de la aleta adiposa $850-880(865)$.

\section{Familia MEGALOPIDAE}

(Fig. 7)

\section{Megalops atlanticus Cuvier \& Valenciennes}

Megalops atlanticus Cuvier \& Valenciennes, 1846: 398, (descripción original; Pequeñas Antillas y Puerto Rico); Regan, 1910: 354, (enumerado; Lago Nicaragua).

Megalops sp. Gill \& Bransford, 1877: 187, (enumerado; rápidos del Toro, Río San Juan. Nicaragua).

Tarpon atlanticus Meek, 1907: 111 (descripción; Lago Nicaragua).

No se obtuvo ningún ejemplar de esta especie; pero en varias ocasiones pude observar algunos ejemplares rompiendo la superficie del lago, cerca de Granada. No hay razón para creer que la población del Lago Nicaragua constituya una forma diferente.

Los caracteres de la especie según Jordan y EvermanN (28) son como sigue: cabeza 4 reces en la longitud del cuerpo; profundidad del cuerpo de 3.8.; D. 
12; A. 20; escamas a lo largo de la línea lateral 42; branquiostegales 23 . Cuerpo alargado, comprimido, poco elevado. El maxilar se extiende más allá de los ojos. Color uniformemente plateado con el dorso más oscuro.

\section{Familia CLUPEIDAE}

(Fig. 8)

\section{Dorosoma chavesi Meek}

Dorosoma chavesi Meek, 1907: 112, (descripción original; Lagos Managua y Nicaragua); Regan, 1908: 177, (enumerado; Nicaragua); Miller, 1950: 398-401, (revisión del género; descripción, claves, relación de $D$. chavesi con otras especies).

Opisthonema libertatis Gill \& Bransford, 1877: 187, (enumerado; Lago Nicaragua).

La descripción que sigue se basa en treinta y seis ejemplares: veinte de 32 a $131 \mathrm{~mm}$ (L. 16); ocho de 124 a $180 \mathrm{~mm}$ (L. 14); cuatro de 91 a $125 \mathrm{~mm}$ (L. 28); dos de $110 \mathrm{~mm}$ cada uno (L. 4); dos de 110 y $122 \mathrm{~mm}$ (L. 3).

Color plateado, azul metálico en el dorso; mancha humeral oscura; aletas de color uniforme. Cabeza grande; boca grande; el maxilar alcanza la vertical trazada desde la mitad del ojo. La longitud de la mandíbula incluida unas dos veces en la de la cabeza. Los ojos grandes y el párpado adiposo bien desarrollado.

D. 11-16; A. 24-30. Pct. 12-15; Plv. 7-8; escamas línea lateral 72-86; escamas alrededor del pedúnculo caudal 19-25; escudetes ventrales prepélvicos 15-18; escudetes ventrales postpélvicos 9-12; filamentos branquiales 110-165, $115-188$.

CUADRO 1

Comparación de ecotipos de Dorosoma chavesi

\begin{tabular}{lccccc}
\hline \multicolumn{1}{c}{ Medida } & \multicolumn{2}{c}{ Lago Managua } & \multicolumn{3}{c}{ Lago Nicaragua } \\
\hline Origen anal - base caudal & $348-369$ & $(359)$ & $364-400$ & $(386)$ \\
Longitud de la cabeza & $326-355$ & $(341)$ & $292-323$ & $(304)$ \\
Profundidad de la cabeza & $258-279$ & $(265)$ & $231-248$ & $(236)$ \\
Morro + ojo & $152-162$ & $(155)$ & $134-148$ & $(141)$ \\
Base aleta anal & $246-271$ & $(263)$ & $276-296$ & $(290)$ \\
Longitud del maxilar & $109-117$ & $(113)$ & $88-100$ & $(90)$ \\
Distancia predorsal & $532-573$ & $(550)$ & $510-545$ & $(530)$ \\
Distancia prepélvica & $492-530$ & $(510)$ & $437-495$ & $(470)$ \\
Ancho del cuerpo & $98-137$ & $(121)$ & $109-132$ & $(122)$ \\
Profundidad del cuerpo & $344-405$ & $(378)$ & $373-389$ & $(379)$ \\
\hline
\end{tabular}

En lo que respecta a las proporciones del cuerpo, Míler (39) encuentra algunas diferencias entre los ejemplares de ambos lagos. Hacemos una comparación entre cinco ejemplares del Lago Managua de 92 a $136 \mathrm{~mm}$ y cinco del Lago Nicaragua de 91 a $125 \mathrm{~mm}$, en lo que respecta a los caracteres arriba citados (Cuadro 1). Los resultados concuerdan con los de Miller si se exceptúan 
la profundidad y el ancho del cuerpo, caracteres en los cuales no se encontró una diferencia apreciable.

Respecto a otros caracteres no citados por Miller no se observó diferencia apreciable entre los ejemplares de ambos lagos. Por esta razón las medidas proporcionales que damos a continuación están basadas en veintiocho ejemplares de la cuenca del Lago Managua: veinte (L. 16); ocho (L. 14).

Longitud estándard 32-180 mm (83); longitud predorsal 507.573 (534); longitud prepélvica 476-547 (517); distancia del origen de la aleta anal al centro de la base de la aleta caudal 312-390 (349); profundidad del cuerpo 294403 (344); ancho del cuerpo 94-137 (116); longitud de la cabeza 299-363 (330); profundidad de la cabeza 232-279 (255); ancho de la cabeza 111-156 (134); distancia interorbital 70.94 (84); longitud del morro 62-81 (72); diámetro de la órbita 67-100 (85); longitud del filamento dorsal 87-174 (133); longitud de la base de la aleta dorsal 105-156 (129); altura de la aleta dorsal 197-240 (219); longitud de la aleta pectoral 144-226 (196); longitud de la base de la aleta anal 213-295 (258); altura de la aleta anal 97-127 (112); longitud de la aleta pélvica 115-148 (125); longitud del lóbulo caudal inferior 260-370 (308); longitud de la mandíbula 125-155 (139); longitud del maxilar 99.126 (112).

\section{Familia POECILIIDAE}

(Figs. 9, 10)

En la descripción de las especies de esta familia se darán primero las medidas de los machos.

\section{CLAVE PARA LOS GENEROS}

1a. Borde inferior del pedúnculo caudal agudo, sin serie de escamas normales ........... Alfaro

1b. Borde inferior del pedúnculo caudal redondeado, con serie de escamas normales.

2a. Sin ensanchamiento membranoso a lo largo del margen anterior del gonopodio. Este último presenta dos apéndices prominentes en forma de cuerno. En las hembras la aleta dorsal comienza algo más adelante que la anal. En los lados del cuetpo aparecen bandas verticales. Xenopballus

2b. Ensanchamiento membranoso a lo largo del margen anterior del gonopodio. Este último sin apéndices prominentes en forma de cuerno. En las hembras la aleta dorsal se origina sobre la anal. No hay bandas verticales a los lados del cuerpo. Mollienesia

\section{Alfaro cultratus Regan}

Petalosoma cultratwm Regan, 1908: 458, (descripción ortginal; Río Iroquois, Costa Rica). 
Nunca había sido reportado en la cuenca de los lagos donde parece ser escaso. La descripción que sigue está basada en dos ejemplares: un macho de $34 \mathrm{~mm}$ (L. 17); una hembra de $35 \mathrm{~mm}$ (L. 5).

Cuerpo muy comprimido, perfil predorsal recto. Boca grande, dientes fijos, la serie externa de dientes más grandes.

D. 8; A. 10; Pct. 14; escamas laterales 32-33; distancia predorsal 648 714; distancia preanal 412-543; distancia prepélvica 368-429; profundidad del cuerpo 264-314; parte más estrecha del pedúnculo caudal 176-171; longitud de la cabeza 264-285; longitud del morro 82-86; diámetro orbital 88; distancia interorbital 94-108; longitud del maxilar 82-86; longitud de la aleta dorsal 264242; longitud de la aleta pectoral 206-256; longitud de la aleta pélvica 185-143; longitud del gonopodio 264; longitud de la aleta anal (hembra) 256.

\section{Xenophallus umbratilis (Meek)}

Gambusia umbratilis Meek, 1912: 70, (descripción original; Guápiles, Costa Rica). Brachyrbaphis umbratilis Meek, 1914: 115, (enumerado; Costa Rica).

La descripción que sigue se basa en veinte ejemplares: cuatro machos de 22 a $23 \mathrm{~mm}$ y cinco hembras de 29 a $33 \mathrm{~mm}$ (L. 1); cuatro machos de 21 a 26 $\mathrm{mm}$ y cinco hembras de 37 a $41 \mathrm{~mm}$ (L. 12); dos machos 22 y $24 \mathrm{~mm}$ (L. 36).

Los machos de esta especie son muy fáciles de identificar por las cinco líneas verticales irregulares, como trazadas con un golpe de lápiz, que aparecen en los costados. Estas líneas aparecen también en las hembras, pero a veces muy difuminadas. La aleta dorsal en los machos presenta dos líneas gruesas, una cerca de la base, y otra en el borde; el espacio entre las dos líneas es de color naranja. Hay también algunas líneas de color naranja a lo largo de las marcas laterales. Las hembras tienen los dos o tres primeros radios de la aleta dorsal negros y esta aleta se origina más cerca de la base de la aleta caudal que del extremo del morro.

Boca pequeña, la mandíbula inferior algo más larga. D. 9-10; A. (hembra) 10; Pct. 10-11; escamas laterales 28-30.

Longitud estándard $21.26 \mathrm{~mm},(23), 29-41 \mathrm{~mm}$ (35); distancia predorsal 500-536 (517), 536-568 (550); distancia preanal 434-478 (458), 567-595 (582); distancia prepélvica 346-386 (372), $433.466 \quad$ (444); profundidad del cuerpo 250-294 (269), 266-306 (277); parte más estrecha del pedúnculo caudal 174-207 (192), 162-193 (172); longitud de la cabeza 230-264 (246), 231-252 (242); longitud del morro 61-82 (70), 67-81 ((72); diámetro de la órbita 81.95 (89), 78.103 (90); distancia interorbital 95-114 (102), 97-115 (105); longitud de la aleta dorsal 233-279 (261), 210-256 (235); longitud de la aleta pectoral 182-218 (207), 178-230 (205); longitud de la aleta pelviana 181-217 (202), 146-188 (165); longitud del gonopodio sin el garfio 326-394 (358); longitud de la aleta anal en las hembras 213-242 (222).

Material: 26 ej. (L. 1); 46 ej. (L. 12); 19 ej. (L. 36). 


\section{Mollienesia Le Sueur}

La identificación de las especies dentro de este género es muy difícil. Se han descrito muchas especies y muchas de ellas muy imperfectamente.

De los lagos han salido descritas por GILL y BRANSFORd (18) Poecilia (Mollienesia) dovii Günther y por Merk (34) Poecilia (Mollienesia) sphenops Cuvier y Valenciennes, como sinónimo de $P$. dovii.

Entre la gran cantidad de Mollienesia colectada podemos reconocer tres especies. Una de ellas corresponde a la descripción dada por Carl L. Hubbs (comunicación personal) para $M$. sphenops después de haber examinado los ejemplares tipo del museo de París. De las otras dos, una podría ser $M$. dovii Günther. Sería necesaria una comparación directa con los tipos para llegar a una conclusión definitiva, dado que las descripciones originales son incompletas.

\section{CLAVE PARA LAS ESPECIES}

1a. Fila interior de dientes ancha, aproximadamente la mitad de la longitud $M$. spbenops

1b. Fila interior de dientes estrecha, menos de una tercera parte de la longitud.

2a. 18 escamas alrededor del pedúnculo caudal

M. dovii?

2b. 16 escamas alrededor del pedúnculo caudal M. $s p$.

\section{Mollienesia sphenops (Cuvier \& Valenciennes)}

Poecilia spbenops Cuvier \& Valenciennes, 1846: 130, (descripción original; Vera Cruz, México); Günther, 1866: 343, (descripción); Meek, 1907: 113, (clasifica todas las Mollienesia de los lagos como M. spbenops); Regan, 1908: 102-103, (sinónimos, descripción; México y Centro América); Jordan \& Evermann, 1896: 694, (descripción; Vera Cruz, México).

Esta especie no parece ser tan abundante en los lagos como las otras dos. La descripción que sigue se basa en ocho ejemplares: cuatro machos de 43 a 46 mm (44) y cuatro hembras de 45 a $55 \mathrm{~mm}$ (48) de (L. 24).

D. 10-11; A. 10-11; Pct. 16; escamas laterales 26; escamas alrededor del pedúnculo caudal 16 .

Distancia predorsal 558-595 (581), 607-624 (616); longitud preanal $470-576$ (525), 624-655 (636); distancia prepélvica $443-489$ (463), 490-510 (498); profundidad del cuerpo $316-337$ (326), 304-328 (313); parte más estrecha del pedúnculo caudal 213-223 (217), 189-200 (195); longitud de la cabeza 256-272 (262), 261-273 (266); longitud del morro 89-98 (94), 89102 (96); diámetro orbital 70-76 (74), 77-89 (81); distancia interorbital 126141 (134), 137-149 (142); longitud de la aleta dorsal 265-326 (299), 222-242 (235); longitud del gonopodio 195-232 (213); longitud de la aleta anal (hembras) $170-200$ (192); longitud de la aleta pectoral 217-232 (223), 200-236 (221). 


\section{Mollienesia dovii (Günther)}

Poecilia dovii Günther, 1866: 344, (descripción original; Lago Nicaragua); Gill \& Bransford, 1877: 187, (enumerado; Lago Nicaragua); Jordan \& Evermann, 1896: 695, (descripción; Lago Nicaragua).

Esta especie parece ser la más abundante en los lagos y probablemente representa la mayor parte de los ejemplares coleccionados por MEEK (34), identificados por él como $M$. sphenops. Esta especie se encuentra juntamente con $M$. sp. a lo largo de las costas de los lagos y se distingue de ésta por su mayor número de escamas en el pedúnculo caudal. Los ejemplares más grandes de la colección pertenecen a esta especie, lo que parece indicar que $M$. dovii alcanza un mayor tamaño que $M$. sp. No se nota diferencia apreciable en lo que respecta a las proporciones del cuerpo.

La descripción que sigue se basa en treinta y cuatro ejemplares: cinco machos de 73 a $91 \mathrm{~mm}$ y cinco hembras de 71 a $95 \mathrm{~mm}$ (L. 3); siete machos de 57 a $97 \mathrm{~mm}$ y nueve hembras de 76 a $118 \mathrm{~mm}$ (L. 14); cuatro machos de 57 a $88 \mathrm{~mm}$ y dos hembras de 72 a $93 \mathrm{~mm}$ (L. 16); una hembra de $100 \mathrm{~mm}$ (L. 28).

D. 10; A. 10-11; Pct. 15-16; escamas laterales 27-30 (28.7); escamas alrededor del pedúnculo caudal 18.

Longitud estándard $57-97 \mathrm{~mm}(80), 71-118 \mathrm{~mm}$ (92); distancia predorsal 540-567 (554), 574-653 (597); distancia preanal 480-561 (507), 612-657 (632); distancia prepélvica 420-494 (448), 476-514 (499); profundidad del cuerpo 308-350 (325), 290-348 (317); parte más estrecha del pedúnculo caudal 221-250 (237), 186-225 (202); longitud de la cabeza 247-274 (264), 259-292 (265); longitud del morro 82-108 (85), 93-111 (101); diámetro de la órbita 69-89 (79), 65-85 (76); distancia interorbital 125-140 (131), 137 161 (143); longitud de la aleta dorsal 252-480 (394), 218-270 (236); longitud de la aleta anal 183-211 (195), 186-208 (195); longitud de la aleta pec. toral 217-268 (245), 206-250 (226).

Material: 2 ej. (L. 28); 8 ej. (L. 16); 6 ej. (L. 4); 44 ej. (L. 3); 1 ej. (L. 13); 111 ej. (L. 6); 30 ej. (L. 36); 30 ej. (L. 14); 2 ej. (L. 31).

\section{Mollienesia sp.}

Esta especie es muy abundante en los lagos y vive juntamente con $M$. dovii. Se le puede distinguir de esta última por su menor número de escamas en el pedúnculo caudal.

En cuanto a la forma del cuerpo no se pudo encontrar diferencia apreciable. Las dos especies se coleccionaron en la localidad (L. 6) donde eran fácilmente distinguibles: $M$. dovii presenta un color amarillo pálido con una mancha negra en la base de la aleta dorsal en ambos sexos; $M$. sp. es de color verdoso con tonos azulados y los machos tienen la característica aleta dorsal grande.

La descripción que sigue se basa en veintitrés ejemplares: un macho de $65 \mathrm{~mm}$ y siete hembras de 66 a $68 \mathrm{~mm}$ (L. 3); un macho de $51 \mathrm{~mm}$ y dos hem. 
REVISTA DE BIOLOGIA TROPICAL

bras de 73 y $78 \mathrm{~mm}$ (L. 17); tres machos de 47 a $69 \mathrm{~mm}$ y cinco hembras de 68 a $88 \mathrm{~mm}$ (L. 31); cuatro machos de 57 a $79 \mathrm{~mm}$ (L. 28).

D. 9-10; A. 9-10; Pct. 15-16; escamas laterales 25-28 (26.4); escamas alrededor del pedúnculo caudal 16.

Longitud estándard $47-79 \mathrm{~mm}$ (59), 66-88 (77); distancia predorsal 540-590 (560), 567-616 (589); distancia preanal 486-598 (533), 598-660 (626); distancia prepélvica 412-521 (462), 476-527 (492); profundidad del cuerpo 316-376 (346), 294-344 (315); parte más delgada del pedúnculo caudal 222-264 (240), 190-221 (207); longitud de la cabeza 254-269 (261), 239274 (256); longitud del morro 77-102 (92), 88-99 (93); diámetro de la órbita 64-91 (79), 52-73 (66); distancia interorbital 128-141 (135), 134-155 (141); longitud de la aleta dorsal 334-456 (387), 235-274 (254); longitud del gonopodio 185-220 (202); longitud de la aleta anal (hembras) 170-208 (186); longitud de la aleta pectoral 222-281 (250), 202-266 (227).

Material: 5 ej. (L. 1); 79 ej. (L. 3); 29 ej. (L. 2); 26 ej. (L. 5); 49 ej. (L. 6); 27 ej. (L. 7); 1 ej. (L. 12); 26 ej. (L. 14); 2 ej. (L. 15); 5 ej. (L. 16); 25 ej. (L. 17); 17 ej. (L. 18); 114 ej. (L. 28); 46 ej. (L. 29); 19 ej. (L. 30); 63 ej. (L. 31); 53 ej. (L. 35); 98 ej. (L. 36).

\section{Familia ATHERINIDAE}

(Fig. 11)

\section{Thyrinops sardina (Meek)}

Melaniris sardina Meek, 1907: 114, (descripción original; Lagos Managua y Nicaragua).

Thyrina sardina Regan, 1908: 189, (enumerado; Nicaragua).

Chirostoma guatemalensis (no el de Günther). Gill \& Bransford, 1877: 187, (enumerado; Nicaragua).

Este pez es muy abundante en los lagos; algunas tardes se le puede ver saltando para agarrar chayules todo a lo largo de la costa de los lagos. Probablemente es uno de los más importantes escalones en la cadena de alimentos.

La descripción que sigue se basa en veinte ejemplares: diez de 45 a 59 mm (L. 16); diez de 41 a 49 mm (L. 36).

El color es blanco amarillento con una banda lateral plateada, la cual comienza estrecha detrás de la parte superior de la inserción de las aletas pectorales, y poco a poco se ensancha hacia la base de la aleta caudal; esta banda es algo más estrecha que la pupila del ojo y ocupa parte de la tercera y cuarta filas longitudinales de escamas (contando desde la base de la aleta dorsal). Hay puntos de un color sucio repartidos a lo largo del dorso, cabeza y aletas impares. La parte superior de la cabeza es casi plana; boca pequeña y muy oblicua; el maxilar alcanza la vertical trazada por la parte anterior de los ojos. El lóbulo cau. dal inferior es un poco más grande que el superior. 
Las escamas son de bordes ligeramente almenados, algunas de borde entero. La aleta anal presenta una lámina de escamas en la primera parte de su base. La línea lateral está interrumpida.

D. 3-4, 9-11; A. 18-22; Pct. 11-14; escamas a lo largo de la línea lateral 35-39; filamentos branquiales 4, 17-19.

Longitud estándard $41-59 \mathrm{~mm}$ (48); distancia predorsal $585-614$ (596); distancia desde el origen del morro hasta el origen de la segunda aleta dorsal 704-754 (722); distancia preanal 550-614 (576); distancia prepélvica 390-428 (407); profundidad del cuerpo 195-231 (212); parte más estrecha del pedúnculo caudal 83-100 (89); longitud de la cabeza 216-250 (234); longitud del morro 54-70 (63); diámetro de la órbita 69-94 (84); distancia interorbital 70-87 (78); longitud del maxilar 69-90 (82); longitud de la base de la aleta anal 257-322 (287); longitud de la aleta pectoral 250-305 (266).

No es claro si hay diferencia entre esta especie y Chirostoma guatemalensis Günther. Siendo muy concisa la descripción (21), sería necesaria una comparación entre los mismos ejemplares.

Material: 89 ej. (L. 1); 71 ej. (L. 3); 7 ej. (L. 4); 47 ej. (L. 5); 1 ej. (L. 6); 47 ej. (L. 7); 75 ej. (L. 12); 100 ej. (L. 13) 13 ej. (L. 16); 2 ej. (L. 28); 87 ej. (L. 29); 15 ej. (L. 30); 5 ej. (L. 31); 19 ej. (L. 36).

\section{Familia POMADASYIDAE}

(Fig. 12)

\section{Ponzadasys grandis Meek}

Pomadasys grandis Meek, 1907: 116, (descripción; Lago Nicaragua); Regan, 1908: 189, (como posible sinónimo de $P$. boucardi).

Pristipoma boucardi Steindachner, 1869: 120, (costa de México). mido.

Sólo se pudo examinar un ejemplar ya abierto y preparado para ser co-

Según Regan (44) $P$. grandis Meek es solamente un sinónimo de $P$. boucardi. Aparentemente $P$. grandis difiere solamente en caracteres que están relacionados con el tamaño, tales como los ojos más pequeños y las espinas más cortas. El único ejemplar examinado por Meek medía $345 \mathrm{~mm}$. Los ejemplares de $P$. boucardi de Regan eran de 170 y $175 \mathrm{~mm}$ de largo.

Los caracteres específicos que separan a $P$. boucardi y $P$. grandis de las otras especies de México y Centro América son: aleta dorsal sin escamas, excepto en su base; segunda espina anal más larga que la tercera; el maxilar no se extiende más allá de la vertical trazada por el margen anterior del ojo; segunda espina anal de 5/6 a $7 / 8$ de longitud de la cabeza; profundidad del cuerpo incluida $31 / 2$ veces en la longitud del mismo.

Será necesaria una comparación con los tipos de $P$. boucardi y colectar más ejemplares para poder confirmar o negar la validez de $P$. grandis. 


\section{Familia CICHLIDAE}

(Fig. 13)

\section{CLAVE PARA LOS GENEROS}

1a. Todos los dientes cónicos o cilíndricos

Cichlasoma

1b. Dientes anteriores comprimidos

2a. Dientes anteriores con bordes enteros Neetroplus

2b. Dientes anteriores con bordes tricúspides Herotilapia

\section{Cicblasoma Swainson}

\section{CLAVE PARA LAS ESPECIES}

1a. Boca grande; la longitud de la mandíbula incluida menos de $21 / 3$ veces en la longitud de la cabeza; en la mandíbula superior los dos dientes anteriores alargados y de aspecto canino; los dientes anteriores de la mandibula inferior pequeños y seguidos por dos dientes de aspecto canino, de los cuales el posterior es más grande.

2a. El suborbital mucho más ancho que el diámetro del ojo. Escamas de la mejilla, de 9 a 11 filas. Escamas en la línea lateral de 35 a 38. C. dovii

2b. El suborbital más estrecho que el diámetro del ojo. Escamas de la mejilla, de 5 a 7 filas. Escamas en la línca lateral de 28 a 31.

3a. Ancho del suborbital incluido de 4 a 5 veces en la longitud del maxilar y más de dos veces en el diámetro de la órbita.

3b. Ancho del suborbital incluido menos de 3 veces en la longitud del maxilar y menos de 2 veces en el diámetro de la órbita.

C. managuense

1b. Boca pequeña; la longitud de la mandíbula incluida más de $21 / 3$ veces en la longitud de la cabeza; dientes subiguales, los anteriores en algunas ocasiones algo más grandes.

4a. Labios anchos y gruesos, prolongados en su parte media en un largo repliegue triangular.

C. labiatum

4b. Labios normales.

5a. Base de la aleta anal de longitud igual o algo mayor que la de la cabeza. Espinas anales de IX a XI.

6a. Ancho del suborbital incluido menos de 2 veces en el diámetro orbital. Longitud de la base de la aleta anal igual a la longitud de la cabeza. Manchas laterales oscuras. Perfil predorsal marcadamente convexo 
6b. Ancho del suborbital incluido 2 o más veces en el diámetro de la órbita. Base de la aleta anal más larga que la cabeza. No presenta manchas laterales oscuras. Perfil predorsal moderadamente convexo. C. centrarcbus

5b. Base de la aleta anal más corta que la cabeza. Espinas de la aleta anal de VI a VIII.

7a. Repliegue del labio inferior contínuo, no interrumpido por un frenillo. C. citrinellum

7b. Repliegue del labio inferior interrumpido por un frenilln.

8a. La aleta pectoral no llega hasta la mitad de la porción espinosa de la aleta anal.

9a. Profundidad del cuerpo incluída 2 o menos veces en la longitud del cuerpo. Aleta caudal redondeada. Mancha caudal grande y redonda. Perfil predorsal no elevado.

C. maculicauda

9b. Profundidad del cuerpo incluido más de 2 veces en la longitud del cuerpo. Aleta caudal emarginada. Sin mancha caudal o con una muy pequeña. Perfil predorsal elevado.

C. nicaraguense

8b. La aleta pectoral pasa de la mitad de la porción espinosa de la aleta anal.

10a. Coloración de los adultos vivos dorado, sin rojo en el vientre. Generalmente sin banda lateral oscura. Ordinariamente tres barras verticales entre la mancha lateral y la base de la aleta caudal. C. rostratum

10b. Coloración de los adultos rojo encendido en el vientre. Generalmente presentan una banda lateral oscura. Ordinaria. mente cuatro barras verticales entre la mancha lateral y la base de la aleta caudal.

C. longimanus

\section{Cichlasoma dovii (Günther)}

Heros dovii Günther, 1864: 154 (descripción original; Lago Nicaragua); Gill \& Bransford. 1877: 183, (enumerado; Nicaragua); Jordan \& Evermann. 1896: 1535, (descripción; Lago Nicaragua).

Cicblasoma dovii Meek, 1907: 120, (descripción; Lagos Managua y Nicaragua); Regan, 1908: 29, (enumerado; Nicaragua).

Esta especie no parece ser tan abundante como $C$. managuense; es un pescado muy bueno como alimento, creciendo hasta un tamaño de $350 \mathrm{~mm}$. Los dos ejemplares más grandes fueron capturados en una zona de poca profundi- 
dad con anzuelo por un nativo que dijo que estos peces eran muy abundantes $y$ fáciles de pescar.

La descripción que sigue se basa en once ejemplares: nueve de 116 a $244 \mathrm{~mm}$ (L. 1); dos de 335 a $348 \mathrm{~mm}$ (L. 13).

Color verdoso oscuro, con fondo azulado; la parte delantera de la mayor parte de las escamas es marrón oscuro; en algunos ejemplares se notan unas siete barras verticales poco visibles; ordinariamente hay una banda lateral muy visible formada por manchas oscuras; desde el ojo a la parte superior del opérculo y a la base de la pectoral se extienden dos bandas oscuras formando los lados de un ángulo; aletas impares manchadas; aletas pares de color uniforme. La boca es grande; el extremo del maxilar alcanza la vertical trazada por la parte anterior del ojo; los labios son gruesos y el repliegue del labio inferior es continuo.

D. XVIII, 11-12; A. VI-VII, 8-10; Pct. 14-15; escamas línea lateral 35-38; escamas de la mejilla 9-11 filas; filamentos branquiales 2-4, 7-9.

Longitud estándard 116-348 mm (200); distancia predorsal 410-437 (419); distancia preanal 672-69.5 (683); distancia prepélvica 416-443 (430); profundidad del cuerpo 352-420 (374); parte más estrecha del pedúnculo caudal 106-155 (134); longitud de la cabeza 387-410 (399); longitud del morro 139168 (155); diámetro de la órbita 64-86 (75); distancia interorbital 76-115 (89); longitud del maxilar 169-198 (179); longitud de la mandíbula 224-252 (237); ancho suborbital 66-94 (77); longitud de la aleta dorsal 640-833 (687); longitud de la aleta anal 353-439 (390); longitud de la aleta pectoral 192-238 (215); longitud de la aleta pelviana 191-250 (224); base de la aleta dorsal 520-572 (535); base de la aleta anal 208-245 (228).

\section{Cichlasoma friedrichstbalii (Heckel)}

Heros friedricbsthalii Heckel, 1840: 381, (descripción original; Río San Juan, Nicaragua); Jordan \& Evermann, 1896: 1528, (descripción; Río San Juan, Nicaragua; Petén, Guatemala).

Cicblasoma friedrichstbalii Regan, 1908: 29, (enumerado; Lago Petén, Guatemala).

Esta especie es muy parecida a $C$. managuense y $C$. dovii, pero se diferencia de éstas por tener la boca mucho más pequeña, el cuerpo más profundo y el margen superior de la aleta dorsal recto.

La siguiente descripción se basa en seis ejemplares: dos de 73 a $84 \mathrm{~mm}$ (L. 2); dos de 85 a $108 \mathrm{~mm}$ (L. 28) y dos de 59 a $84 \mathrm{~mm}$ (L. 36).

Color de los ejemplares vivos oscuro, con tonos verdes; bandas verticales borrosas más marcadas en su parte media, formando así una banda lateral; manchas oscuras en la base de la cola; aletas dorsal, anal, y caudal con dibujos como las vetas del mármol; aletas pares lisas; dos manchas negras en el opérculo; una línea negra desde el borde de la órbita hasta la mancha opercular inferior. 
D. XVII-XVIII, 9-10; A. IX, 8-9; Pct. 13-14; escamas línea lateral 28-30; filas de escamas en la mejilla 5-7; filamentos branquiales 3, 9-10.

Longitud estándard 59-108 mm (82); distancia predorsal 397-435 (416); distancia preanal 620-647 (637); distancia prepélvica 381-432 (420); profundidad del cuerpo 408-440 (428); parte más estrecha del pedúnculo caudal 136-165 (151); longitud de la cabeza 388-397 (393); longitud del morro 119131 (124); diámetro de la órbita 85-115 (100); distancia interorbital 102-112 (107); longitud del maxilar 132-143 (139); ancho suborbital 48-55 (52); longitud de la aleta dorsal 710-865 (784); longitud de la aleta anal 510-566 (530); longitud de la aleta pectoral 269-288 (274); longitud de la aleta pelviana 234318 (294); base de la aleta dorsal 575-595 (580); base de la aleta anal 315 . 322 (319).

\section{Cichlasoma managuense (Günther)}

Heros managuensis Günther, 1869: 463, (descripción original, dibujos; Lagos Managua y Nicaragua); Jordan \& Evermann, 1896: 1533, descripción; Lago Managua).

Cicblasoma mallaguense Meek, 1907: 119, (descripción; Lagos Managua y Nicaragua); Rc gan, 1908: 29, (enumerado; Nicaragua).

Esta especie es muy abundante y parce ser tan valiosa como $C$. dovii como alimento. Aún en lugares fangosos y de poca profundidad se encontraron pequeños ejemplares juntamente con otros cíclidos. Se le encuentra también en grandes cantidades en la Laguna de Apoyo (L. 6) donde tal vez debido a la cscasez de alimento los ejemplares son mucho más delgados.

Hay en la colección un ejenplar que parcce pertenecer a csta especie, pero tenía en vida un hermoso color dorado con algunos puntos oscuros poco marcados en las mejillas y parte anterior del cuerpo. El borde inferior de la aleta caudal, extremos de las espinas de la aleta anal y el borde exterior de las aletas pelvianas son ncgros. Este ejemplar fue conseguido para la colección por el Sr. Camacho. Por ser tan raros, dicen los pescadores que sólo los pescan una o dos veces en toda la vida. Los nativos lo llaman "Rey de los guapotes" y creen que si este pez es comido crudo tan pronto como es sacado del agua les traerá buena suerte en la pesca durante todo el año; si lo dejan ir o lo venden en el mercado, les traerá mala suerte en la pesca.

Después de un cuidadoso examen comparativo con ejemplares de $C$. managuense, teniendo también en cuenta su extrema rareza, se puede suponer que el "Rey de los guapotes" es un ejemplar albino de C. managuense.

La descripción que sigue se basa en veinte ejemplares: ocho de 30 a $97 \mathrm{~mm}$ (L. 31); tres de 185 a $218 \mathrm{~mm}$ (L. 16); dos de 61 y $65 \mathrm{~mm}$ (L. 3); dos de 38 y $48 \mathrm{~mm}$ (L. 28); dos de 29 y $51 \mathrm{~mm}$ (L. 30); uno de $108 \mathrm{~mm}$ (L. 1); uno de $35 \mathrm{~mm}$ (L. 12); uno de $175 \mathrm{~mm}$ (L. 36).

Los dientes y la coloración son como en $C$. dovit; pero $C$. managuense se puede distinguir fácilmente por tener el suborbital mucho más estrecho y un menor número de filas de escamas en las mejillas. 
D. XVII, 10-12; A. VI-VIII, 8-9; Pct. 13-15; escamas línea lateral 31-35 (33); filas de escamas en la mejilla 5-7; filamentos branquiales 2-3, 8-12.

Longitud estándard 29-218 mm (79); distancia predorsal 404-453 (431); distancia preanal 629-676 (658); distancia prepélvica 414.470 (449); profundidad del cuerpo 361-412 (385); parte más estrecha del pedúnculo caudal 129-153 (142); longitud de la cabeza 383-449 (421); longitud del morro 100-131 (114); diámetro de la órbita 74-138 (108); distancia interorbital 84-109 (94); longitud del maxilar 137-187 (161); longitud de la mandíbula 172-248 (211); ancho suborbital 26.45 (33); longitud de la aleta dorsal 639-715 (680); longitud de la aleta anal 394-454 (429); longitud de la aleta pectoral 236-300 (267); longitud de la aleta pelviana 224-302 (269); base de la aleta dorsal 500-580 (538); base de la aleta anal 249-292 (270).

Material: 1 ej. (L. 1); 2 ej. (L. 3); 68 ej. (L. 6); 5 ej. (L. 12); 2 ej. (L. 28); 1 ej. (L. 29); 2 ej. (L. 30); 10 ej. (L. 31); 19 ej. (L. 36).

\section{Cichlasoma labiatum (Günther)}

Heros labiatus Günther, 1864: 27, (descripción original, dibujo; Lago Nicaragua); Gill \& Bransford, 1877: 182, (enumerado; Nicaragua); Jordan \& Evermann, 1896: 1530, (descripción; Lagos Nicaragua y Managua).

Cicblasoma labiatum Meek, 1907: 124, (descripción; Lago Nicaragua); Regan, 1908: 26, (enumerado; Nicaragua).

? Heros lobocbilus Günther, 1869: 457, (descripción, dibujo; Lago Managua).

? Cicblasoma lobochilus Meek, 1907: 125, (descripción; Lagos Managua y Nicaragua); Regan, 1908: 25, (enumerado; Nicaragua).

La descripción que sigue se basa en diecisiete ejemplares de 117 a 177 $\mathrm{mm}$ de longitud (L. 1). La característica más notable de esta especie son los labios gruesos y sobresalientes que forman una especie de repliegue triangular en ambas mandíbulas.

La coloración es muy variada. La escala de colores corresponde a la de C. citrinellum. La mayor parte de los ejemplares son negros pero hay uno rojo y dos rosados. Todos son semejantes en lo que respecta a proporciones y nú. meros.

D. XVI-XVII, 11-12; A. VI-VII, 7-10; Pct. 14-15; escamas línea lateral 30-34; filas de escamas en la mejillas 4; filamentos branquiales 2.5, $10 \cdot 14$.

Longitud estándard $117-177 \mathrm{~mm}$ (139); distancia predorsal $429-449$ (436); distancia preanal 658-719 (691); distancia prepélvica 435-486 (448); profundidad del cuerpo 429-469 (442); parte más estrecha del pedúnculo caudal 135-156 (144); longitud de la cabeza 364-405 (383); longitud del morro 146 166 (154); diámetro de la órbita 82-101 (88); distancia interorbital 107-148 (125); longitud del maxilar 123-151 (137); ancho suborbital 93-110 (100); longitud de la aleta dorsal 664-815 (742); longitud de la aleta anal 379.474 (417); longitud de la aleta pectoral 271-315 (293); longitud de la aleta pél- 
vica 240-326 (291); base de la aleta dorsal 540-584 (561); base de la aleta anal $237-271$ (258).

Dado que ambas especies muestran las mismas variantes de color, se seleccionaron para ser comparadas dos series de C. citrinellum y C. labiatum, incluyendo ejemplares de tamaño semejante. Se observaron diferencias constantes; C. labiatum tiene más cortas las bases de las aletas dorsal y anal. Las aletas pectorales y pélvicas son más cortas y el subordital más estrecho. La cabeza es más larga. Por lo tanto tal vez $C$. citrinellum sea una especie válida y lo mismo C. labiatum.

La especie nominal C. lobocbilus parece ser un sinónimo de $C$. labiatum. Las diferencias dadas por MEeK (34), REgan (44) y Jordan \& EverMaNN (28) se basan en la coloración o son medidas que claramente se superponen. Como no hay en el material de la colección ninguna diferencia marcada entre los ejemplares con labios gruesos, se supone que solo hay una especie con labios gruesos en los lagos.

\section{Cichlasoma spilurum (Günther)}

Heros spilurus Günther, 1862: 289, (descripción original; Lago Izabal, Guatemala); Jordan \& Evermann, 1896: 1520, (descripción; Río Motagua, Guatemala).

Cicblasoma septemfasciatus Regan, 1908: 461, (Rio Iroquois, Costa Rica).

Cicblasoma spilurum Meek, 1914: 126, (descripción; ambas vertientes, Costa Rica).

La descripción que sigue se basa en veinte ejemplares: diez de 31.5 a $61 \mathrm{~mm}$ (L. 18) y diez de 43 a $69 \mathrm{~mm}$ (L. 35).

Color muy oscuro; algunos de los ejemplares, especialmente los de las aguas más frías del río Grande de Matagalpa, tenian el vientre de color naranja; siete a ocho bandas verticales; la primera y la segunda inclinadas hacia adelante; la segunda y la tercera convergen en el medio formando un dibujo en forma de $\mathrm{V}$ detrás de la cabeza; algunas veces la tercera banda es corta, reducida a una mancha alargada debajo del origen de la aleta dorsal; la segunda, la cuarta y la quinta bandas son más oscuras en el medio; hay una mancha caudal oscura.

Dientes de forma canina, con puntas de color castaño, inclinados ligeramente hacia atrás; fila externa formada por dientes más grandes; boca pequeña; el maxilar no se extiende hasta la vertical trazada por el margen anterior del ojo.

Perfil predorsal elevado; aleta caudal redondeada; parte blanda de aletas dorsal y anal a veces aparece ensanchada y prolongda casi hasta el extremo de la aleta caudal; aletas con finos puntitos como de polvo; aletas pélvicas y pectorales alcanzan el origen de la aleta anal.

D. XVII-XVIII, 8-10; A. IX-X, 7-8; Pct. 12-13; escamas línea lateral 25-29; filas de escamas en la mejilla 4-5; filamentos branquiales 1-3, 5-8.

Longitud estándard 31.5-69 mm (51); distancia predorsal 403.439 (423); distancia preanal 590-635 (614); distancia prepélvica 390-430 (409); 
parte más estrecha del pedúnculo caudal 139-164 (152); longitud de la cabeza 346-396 (66); longitud del morro 101-149 (127); diámetro de la órbita 100-127 (110); distancia interorbital 105-132 (107); longitud del maxilar 91-137 (106); ancho del suborbital $54-87$ (72); longitud de la aleta dorsal 760-910 (833); longitud de la aleta anal 525-695 (574); longitud de la aleta pectoral 290-333 (315); longitud de la aleta pelviana 302-439 (342); base de la aleta dorsal 594-655 (628); basa de la aleta anal 348-385 (365).

Los ejemplares encajan con la descripción de C. spilurum y también con Ia descripción de $C$. nigrofasciatum, excepto por el hecho de que $C$. nigrofasciatum se supone que tiene los dientes cónicos algo aplanados. Como algunos de nuestros ejemplares, que por lo demás son idénticos a los otros, muestran un ligero aplanamiento de la fila exterior de dientes, pudiera ser que las dos especies fueran la misma.

Material: 2 ej. (L. 1); 1 ej. (L. 2); 4 ej. (L. 3); 2 ej. (L. 5); 2 ej. (L. 16); 2 ej. (L. 17); 22 ej. (L. 18); 7 ej. (L. 24); 16 ej. (L. 28); 1 ej. (L. 29); 1 ej. (L. 30); 5 ej. (L. 31); 10 ej. (L. 35); 15 ej. (L. 36).

\section{Cicblasoma contrarchus (Gill \& Bransford)}

Heros centrarcbus Gill \& Bransford, 1877: 185. (descripción original; Lago Nicaragua).

Cicblaroma centrarchuls Jordan \& Evermann, 1896: 1526, (descrinción; Lago Nicaragua); Meek, 1907: 126, (descripción; Iagos Managua y Nicaragua); Regan, 1908: 23, (cnumerado, dibujo; Lago Nicaragua).

La siguiente descripción se basa en veinte ejemplares: sicte de 74 a 100 mm (L. 16); seis de 31 a $54 \mathrm{~mm}$ (L. 5); seis de 45 a $87 \mathrm{~mm}$ (L. 4); uno de $45 \mathrm{~mm}$ (L. 3).

Coloración muy variable, de plateado con bandas verticales muy borro. sas hasta casi negro; ordinariamente siete bandas verticales, la última generalmente más oscura, formando juntamente con una banda oscura en la base de la aleta caudal una especie de mancha caudal doble; dos manchas oscuras en los opérculos. Cuerpo comprimido y alto; aletas pélvicas alcanzan la segunda o tercera espina anal; origen de la aleta anal debajo del primer tercio de la base de la dorsal; aleta caudal redondeada.

Dientes de la fila externa cónicos, con puntas de color naranja y formando una serie regular; dientes internos pequeños, en unas tres filas.

D. XV-XVIII, 8-10; A. X-XI, 8-10; Pct. 13; escamas línea lateral 27-30; filas de escamas en las mejillas de 3 a 5; filamentos branquiales 5-10, 15-20.

Longitud estándard 31-100 mm (63); distancia predorsal 424-474 (457); distancia preanal 550-610 (584); distancia prepélvica 412-460 (431); profundidad de! cuerpo 460-554 (511); parte más estrecha del pedúnculo caudal 131-175 (159); longitud de la cabeza 366-421 (387); longitud del morro 95-120 (109); diámetro de la órbita 94-144 (118); distancia interorbital 108153 (130); longitud del maxilar 95-130 (113); ancho suborbital 38-60 (48); longitud de la aleta dorsal 720-876 (793); longitud de la aleta anal 555-704 
(611); longitud de la aleta pectoral 316-368 (342); longitud de la aleta pélvica 304.378 (333); base de la aleta dorsal 557-623 (591); base de la aleta anal $394-435$ (411).

Material: 2 ej. (L. 1); 15 ej. (L. 3); 33 ej. (L. 4); 5 ej. (L. 7); 5 ej. (L. 14); 7 ej. (L. 28); 9 ej. (L. 30); 5 ej. (L. 36).

\section{Cicblasoma citrinellum (Günther)}

Heros citrinellus Günther, 1864: 153, (descripción original; Lagos Managua y Nicaragua); Gill \& Bransford, 1877: 182, (enumerado; Lago Nicaragua); Jordan \& Evermann, 1896: 1534, (descripción; Lago Nicaragua).

Cichlasoma citrinellum Meek, 1907: 121, (descripción; Lagos Managua y Nicaragua); Regan, 1908: 25, (enumerado; Lagos Managua y Nicaragua).

? Heros erytbraeus Günther, 1896: 457, (dibujo; Lago Managua); Jordan \& Evermann, 1896: 1531, (descripción; Lago Managua).

? Cichlasoma erytbraeum Meek, 1907: 124, (descripción; Lago Managua); Regan, 1908: 25 (enumerado; Lago Managua).

Hay en la colección ejemplares rojos, rosados y negros y algunos oscuros con bandas verlicales muy poco marcadas. Los ejemplares de distinto color no muestran otra diferencia. Por lo tanto se supone que se trata de una sola es. pecie con diferentes colores, y que $C$. erythraeum es la variedad roja de $C$. ci. trinellum. Todos nuestros ejemplares de colores vivos fueron obtenidos en agua limpia. En los ríos pequeños, de fondo fangoso, sólo se obtuvieron ejemplares pequeños, ninguno de ellos con colores vivos; hay datos de ejemplares pequeños rojos conservados en acuarios en Granada.

$\mathrm{La}$ descripción que sigue se basa en diecisiete ejemplares de 127 a 169 $\mathrm{mm}$ de longitud (L. 1).

D. XVI-XVII (16.9), 10-12 (11.8), A. VI-VIII (7), 8.9 (8.8); Pct. $14-15$ (14.5); escamas línea lateral 30-34 (32.5); filas de escamas en las mejillas de 3 a 5 (4); filamentos branquiales $2 \cdot 4,8-11$.

Longitud estándard 127-169 mm (137); distancia predorsal 406-466 (429); distancia preanal 620-665 (644); distancia prepélvica 408-439 (422); profundidad del cuerpo 394-514 (481); parte más estrecha del pedúnculo caudal 138155 (146); longitud de la cabeza 353-380 (366); longitud del morto 132.158 (146); diámetro de la órbita 83-101 (92); distancia interorbital 134-166 (145); longitud del maxilar 116-132 (124); ancho suborbital 79-105 (94); longitud de la aleta dorsal 767-920 (852); longitud de la aleta anal 482-572 (520); longitud de la aleta pectoral 310-359 (338); longitud de la aleta pelviana 282-352 (328); base de la aleta dorsal 574-656 (619); base de la aleta anal 264-322 (292).

Material: 16 ej. (L. 3); 26 ej. (L. 4); 2 ej. (L. 5); 22 ej. (L. 6); 18 ej. (L. 7); 9 ej. (L. 12); 28 ej. (L. 13); 5 ej. (L. 14); 13 ej. (L. 16); 13 ej. (L. 17); 2 ej. (L. 18); 7 ej. (L. 28); 9 ej. (L. 29); 10 ej. (L. 30); 7 ej. (L. $31) ; 28$ ej. (L. 36). 


\section{Cicblasoma maculicauda Regan}

Cicblasoma maculicauda Regan, 1905: 227, (descripción original; Lago Izabal, Río Motagua, Guatemala; Río Chagres, Panamá).

Esta especie parece ser más abundante en los rios de la vertiente atlántica, donde se recolectaron siete ejemplares. Se vieron algunos cíclidos saltando por encima de las redes, a veces hasta unos dos pies de altura, durante la expedición al arroyo "Dead Man's Creek" en la costa atlántica (Blue Fields), que parecían pertenecer a esta especie. Solo dos se colectaron en la cuenca de los lagos.

La descripción que sigue se basa en dos ejemplares: uno de $148.5 \mathrm{~mm}$ ( $\mathrm{L}$. 1); uno de $31 \mathrm{~mm}$ (L. 12).

Color castaño, muy oscuro en el ejemplar más grande; mancha negra grande en la base de la aleta caudal, lo cual hace que esta especie sea muy fácil de separar de cualquier otro cíclido que viva en la cuenca de los lagos. En el ejemplar pequeño son escasamente visibles siete bandas verticales; aletas oscuras, exceptuando las pectorales que no tienen color.

Cuerpo comprimido y muy profundo; cabeza corta, boca muy pequeña; el extremo del maxilar no alcanza la vertical trazada por la parte anterior de la orbita; aleta caudal redondeada con algunos puntos en su tercio basal; margen superior de la aleta dorsal recto; bases de las aletas dorsal, anal y caudal escamosas.

D. XVII, 12-13; A. VI, 9-10; Pct. 15; escamas línea lateral 33-34; filas de escamas en la mejilla 4; filamentos branquiales I, 3-8.

Longitud estándard 31-148.5 (89); distancia predorsal 430-484 (457); distancia preanal 680-710 (690); distancia prepélvica 418-452 (435); profundidad del cuerpo 500-548 (524); parte más estrecha del pedúnculo caudal 168. 177 (172); longitud de la cabeza 356-386 (371); longitud del morro 129-141 (135); diámetro orbital 94-135 (114); distancia interorbital 129-141 (135); longitud del maxilar 97-108 (102); ancho suborbital 65-94 (79); longitud de la aleta dorsal 775-900 (837); longitud de la aleta anal 452-512 (482); longitud de la aleta pectoral 290-298 (294); longitud de la aleta pelviana 322-357 (339); base de la aleta dorsal 580-613 (596); base de la aleta anal 274-280 (277).

\section{Cicblasoma nicaraguense (Günther)}

Heros nicaraguensis Günther, 1864: 153, (descripción original; Lago Nicaragua); Jordan \& Evermann, 1896: 1532, (descripción; Lago Nicaragua).

Cichlasoma nicaraguense Meek, 1907: 129, (descripción; Lagos Managua y Nicaragua); Regan, 1908: 22, (enumerado; Lago Nicaragua).

? Heros balteatus Gill \& Bransford, 1877: 184, (descripción; Lago Nicaragua).

? Cichlasoma balteatum Jordan \& Evermann, 1896: 1521, (descripción; Lago Nicaragua); Meek, 1907: 129, (descripción, posible sinónimo de C. nicaraguesjs; Lagos Managua y Nicaragua); Regan, 1908: 22, (enumerado, dibujo; Lago Nicaragua). 
La descripción y discusión siguiente se basa en veintiseis ejemplares: cinco de 120 a $135 \mathrm{~mm}$ (L. 1); diez de 73 a $114 \mathrm{~mm}$ (L. 36); cuatro de 45 a 110 mm (L. 30); tres de 27 a $40 \mathrm{~mm}$ (L. 12); uno de $64 \mathrm{~mm}$ (L. 5); uno de 70 mm (L. 18); uno de $72 \mathrm{~mm}$ (L. 28); uno de $35 \mathrm{~mm}$ (L. 29).

D. XVIII-XX, 8-11; A. VII-VIII, 7-8; Pct. 12-14; escamas línea lateral 33-38; filas de escamas en la mejilla 4-5; filamentos branquiales 2-3, 6-9.

Longitud estándard 27-135 mm (81); distancia predorsal 382-429 (411); distancia preanal 570-650 (619); distancia prepélvica 356.412 (380); profundidad del cuerpo 389-455 (418); parte más estrecha del pedúnculo caudal 118-141 (131); longitud de la cabeza 319-370 (331); longitud del morro 104-171 (133); diámetro de la órbita 94-135 (111); distancia interorbital 78-117 (106); longitud del maxilar 78-117 (97); ancho suborbital 37-119 (85); longitud de la aleta dorsal 710-910 (801); longitud de la aleta anal 412.584 (483); longitud de la aleta pectoral 254-325 (295); longitud de la aleta pelviana 276-384 (311); base de la aleta dorsal 542-620 (597); base de la aleta anal 250-298 (277).

Hay alguna diferencia, debida a la edad, en la coloración del cuerpo y forma de la cabeza entre los ejemplares colectados. Por esta razón se escogieron y examinaron separadamente tres serias ontogenéticas, con los resultados si. guientes: los ejemplares más pequeños, de 27 a $78 \mathrm{~mm}$ de longitud estándard, presentan una mancha lateral muy bien marcada y banda lateral muy borrosa. Las aletas impares están ligeramente punteadas. Los ejemplares de tamaño medio, de 79 a $114 \mathrm{~mm}$, tienen la banda lateral mejor marcada y la mancha lateral es relativamente más pequeña, haciéndose más pequeña al aumentar el tamaño del ejemplar; las aletas son punteadas. Estos ejemplares de tamaño medio fueron pescados a mano; parecían estar vigilando unos agujeros en el barro del fondo (L. 36) en los cuales se escondían rápidamente cuando se les molestaba. Los ejemplares más grandes, de 120 a $135 \mathrm{~mm}$, son mucho más oscuros, la banda y la mancha laterales son muy borrosas y las aletas impares están marcadamente punteadas. Hay una clara gradación de color entre estos tres grupos. No se pudo determinar el sexo de los ejemplares más grandes, porque las gónadas eran pequeñas.

No hay diferencia en lo que respecta a los números de escamas, etc. Hay diferencia en algunas de las medidas de la cabeza; pero se puede observar una clara gradación, lo cual hace que estas diferencias puedan ser atribuidas al crecimiento. (Cuadro 2).

\section{CUADRO 2}

Medidas comparativas de tres series de Cichlasoma nicaraguense Gunther

\begin{tabular}{lccc}
\hline & 27 a $74 \mathrm{~mm}$ & 79 a $114 \mathrm{~mm}$ & 120 a $135 \mathrm{~mm}$ \\
\hline Longitud morro & $104-143$ & $126-149$ & $149-171$ \\
Diámetro órbita & $108-135$ & $101-114$ & $94-100$ \\
Distancia interorbital & $74-114$ & $101-112$ & $133-141$ \\
Longitud maxilar & $78-100$ & $92-107$ & $102-117$ \\
Ancho suborbital & 37.80 & $77-91$ & $100-119$ \\
\hline
\end{tabular}


Hay también un aumento regular en la longitud de las aletas impares. Los dientes son iguales en todos los ejemplares; la fila externa es regular, formada por pequeños dientes cónicos algo aplanados y con los extremos de color yodo.

En los ejemplares más grandes el perfil de la cabeza es muy elevado y casi vertical. Los huesos suborbitales se agrandan dando al pez un aspecto muy característico.

\section{Cicblasoma rostratum (Gill \& Bransford)}

Heros rostratus Gill \& Bransford, 1877: 181, (descripción original; Lago Nicaragua).

Cichlasoma rostratum Jordan \& Evermann, 1896: 1522, (descripción; Lago Nicaragua); Meek, 1907: 127, (descripción; Lagos Managua y Nicaragua); Regan, 1908: 25, (enumerado, figura; Lago Nicaragua).

La siguiente descripción y discusión se basa en veinte ejemplares: ocho de 53 a $120 \mathrm{~mm}$ (L. 4); cinco de 107 a $171 \mathrm{~mm}$ (L. 14); tres de 77 a $109 \mathrm{~mm}$ (L. 18); tres de 50 a $95 \mathrm{~mm}$ (L. 36); uno de $104 \mathrm{~mm}$ (L. 30).

D. XV-XVII, 10-12. A. VI-VII, 7-9; Pct. 14-15; escamas línea lateral 30-34; filas de escamas en la mejilla 4-5; filamentos branquiales 4-5, 11-15.

Longitud estándard 50-171 mm (98); distancia predorsal 420-540 (484); distancia preanal 650-694 (671); distancia prepélvica 420-458 (436); profundidad del cuerpo 446-544 (486); parte más estrecha del pedúnculo caudal 131-159 (145); longitud de la cabeza 382-432 (408); longitud del morro 151-215 (184); diámetro de la órbita 99-141 (116); distancia interorbital 95-134 (115); longitud del maxilar 110-147 (128); ancho suborbital 80-134 (102); longitud de la aleta dorsal 697-800 (745); longitud de la aleta anal 400-480 (442); longitud de la aleta pectoral 302-434 (380); longitud de la aleta pélvica 383-396 (329); base de la aleta dorsal 527-606 (557). Base de la aleta anal 238-290 (257).

En los ejemplares grandes, la coloración general, en vida, es dorada o cobre; bandas borrosas; mancha redonda oscura debajo de la línea lateral en la vertical trazada desde las primeras cuatro espinas anales; cabeza más oscura que el cuerpo, los labios ordinariamente casi negros; mancha caudal muy borrosa o ausente; aleta caudal y partes blandas de las dorsal y anal manchadas de blanco, formando un dibujo como de tablero de damas; aletas pelvianas oscuras y pectorales incoloras.

Dientes pequeños, cónicos con puntas redondeadas de color castaño; los de la fila exterior algo más largos.

En la mayor parte de los ejemplares adultos, la forma de la cabeza es muy caracteristica, estando estirada hacia adelante de tal forma que la distancia desde la punta del morro hasta la órbita es mayor que la distancia desde ésta hasta la parte posterior del opérculo; labios gruesos.

Los ejemplares más pequeños son muy difíciles de separar de los de $C$. longimanus. En ejemplares que por otros datos podrían ser identificados como de esta especie o de $C$. longimanus se encuentran al azar aletas lisas o manchadas. 
El manchón lateral parece darse siempre en ejemplares jóvenes de ambas especies. Esto llevó a Meek (34) a pensar que tal vez $C$. longimanus y $C$. rostratum eran sexos diferentes de la misma especie.

Cinco de nuestros ejemplares más grandes de $C$. rostratum resultaron ser hembras pero de cinco ejemplares grandes de $C$. longimanus, tres eran machos y dos hembras. Esto parece indicar que se trata de dos especies diferentes aunque muy próximas.

En los ejemplares pequeños de $C$. rostratum el morro es más puntiagudo, los lóbulos caudales cortados más limpiamente y generalmente presentan sólo tres bandas verticales entre la mancha lateral y la mancha caudal. En los ejemplares pequeños de $C$. Longimanus, la segunda banda vertical después de la mancha lateral está ordinariamente dividida en dos bandas estrechas.

Material: 11 ej. (L. 4); 1 ej. (L. 5); 18 ej. (L. 12); 5 ej. (L. 14); 6 ej. (L. 18); 7 ej. (L. 28); 1 ej. (L. 29); 1 ej. (L. 30); 1 ej. (L. 31); 8 ej. (L. 36$)$.

\section{Cichlasoma longimanus (Günther)}

Heros longimanus Günther, 1869: 453, (descripción original, figura; Lago Nicaragua); Gill \& Bransford, 1877: 182, (enumerado; Lago Nicaragua); Jordan \& Evermann, 1896: 1521, (descripción; Lago Nicaragua).

Cichlasoma longimanus Meek, 1907: 127, (descripción; Lagos Managua y Nicaragua); Regan, 1908: 24, (enumerado; Lago Nicaragua).

Esta especie es unuy próxima a $C$. rostratum como se ha indicado al hablar de esta última especie.

La siguiente descripción se basa en veinte ejemplares: ocho de 53 a 114 mm (L. 14); seis de 59 a $96 \mathrm{~mm}$ (L. 3); tres de 54 a $88 \mathrm{~mm}$ (L. 4); dos de 55 y $78 \mathrm{~mm}$ (L. 16); uno de $97 \mathrm{~mm}$ (L. 36).

color castaño.

Dientes pequeños, cónicos; la fila externa con puntas redondeados de

D. XVI-XVII, 9-11; A. VI-VII, 7-9; Pct. 13; escamas línea lateral 29-33; filas de escamas en las mejillas 3-4; filamentos branquiales 3-5, 11-15.

Longitud estándard 53-114 mm (83); distancia predorsal 447.495 (471); distancia preanal 624-682 (660); distancia prepélvica 406-444 (426); profundidad cel cuerpo 457-562 (507); parte más estrecha del pedúnculo caudal 148174 (159); longitud de la cabeza 372-408 (391); longitud del morro 132-177 (158); diámetro de la órbita 103-138 (121); distancia interorbital 110-135 (119); longitud del maxilar 110-132 (119); ancho suborbital 75-117 (93); longitud de la aleta dorsal 755-842 (782); longitud de la aleta anal 458-500 (473); longitud de la aleta pectoral 382-453 (420); longitud de la aleta pelviana 294-388 (339); base de la aleta dorsal 552-623 (587); base de la aleta anal 260.302 (281).

Esta especie se encuentra siempre en aguas fangosas y poco profundas. Los niños los agarran con la mano entre las plantas de las orillas. Ios ejempla. 
res grandes, al ser sacados del agua, son muy fáciles de distinguir por su vien. tre de color rojo vivo. El resto del cuerpo es claro, con bandas verticales oscuras, ordinariamente muy oscuras en el centro, las cuatro primeras forman una banda lateral interrumpida; hay ordinariamente una banda oscura que va desde el ojo hasta la primera mancha negra de la banda lateral; aletas manchadas en algunos ejemplares; pelvianas oscuras y pectorales incoloras.

El morro no es tan puntiagudo como el de C. rostratum y los labios son nor. males. Algunos ejemplares, después de preservados, parecen intermedios entre $C$. rostratum y $C$. Longimanus, aunque en vida fueran fácilmente separables por su coloración.

Material: 18 ej. (L. 3); $14 \mathrm{ej.} \mathrm{(L.} \mathrm{4);} 1$ ej. (L. 7); 15 ej. (L. 14); 6 ej. (L. 16); 11 ej. (L. 17); 2 ej. (L. 18); 8 ej. (L. 28); 3 ej. (L. 29); 6 ej. (L. 30); 16 ej. (L. 31); 2 ej. (L. 36).

\section{Neetroplus nematopus Günther}

Neetroplus nematopus Günther, 1869: 464, (descripción original; Lagos Managua y Nicaragua); Regan, 1908: 31, (descripción; Lagos Managua y Nicaragua).

Neetroplus nematopsis Meek, 1907: 130, (descripción; Lago Managua).

Neetroplus nicaraguensis Gill \& Bransford, 1877: 186, (descripción; Lago Nicaragua); Jordan \& Evermann, 1896: 1542, (descripción; Lago Nicaragua).

La siguiente descripción está basada en veinte ejemplares: siete de 42 a $74 \mathrm{~mm}$ (L. 36); cuatro de 39 a $53 \mathrm{~mm}$ (L. 1); tres de 55 a $71 \mathrm{~mm}$ (L. 4); uno de $90 \mathrm{~mm}$ (L. 3); uno de $35 \mathrm{~mm}$ (L. 5); uno de $50 \mathrm{~mm}$ (L. 12); uno de 82 mm (L. 14); uno de $43 \mathrm{~mm}$ (L. 15).

Color castaño oscuro, con bandas verticales muy borrosas; una banda más oscura, especialmente en los ejemplares más pequeños, desde la mitad de la base de la aleta dorsal hasta la mitad del cuerpo. Cola truncada, algunos ejemplares muestran una cresta nucal. Boca muy pequeña. Perfil de la cabeza elevado; aletas pectorales cortas, no llegando al origen de la aleta anal; aletas pelvianas, dorsal y anal prolongadas en un filamento en los ejemplares más grandes; base de las aletas impares escamosa.

Dientes comprimidos, de forma de incisivos, formando una linea regular en cada mandíbula. Los dientes anteriores, seis o siete en cada lado, cuadrados y de un color rojo yodo; unos pocos dientes inferiores en cada mandíbula.

D. XVIII-XIX, 9-11; A. VI-VIII, 6-8; Pct. 13-15; escamas línea lateral 31-35; filas de escamas en la mejilla 4-5; filamentos branquiales 2-4, 7-9.

Longitud estándard $35.90 \mathrm{~mm}$ (55); distancia predorsal 368.405 (385); distancia preanal 628-683 (654); distancia prepélvica 358-392 (374); profundidad del cuerpo $385-452$ (417); parte más estrecha del pedúnculo caudal 120-146 (134); longitud de la cabeza 306-346 (327); longitud del morro 127-158 (141); díámetro de la órbita 85-122 (101); distancia interorbital 91 122 (103); longitud del maxilar 72-100 (83); ancho suborbital 73-122 (92); 
longitud de la aleta dorsal 743-965 (813); longitud de la aleta anal 420-571 (468); longitud de la aleta pectoral 236-291 (264); longitud de la aleta pélvica 274-439 (330); base aleta dorsal 570-651 (622); base aleta anal 233-280 (257).

Material: 9 ej. (L. 1); 1 ej. (L. 3); 3 ej. (L. 4); 5 ej. (L. 5); 1 ej. (L. 7); 1 ej. (L. 12); 1 ej. (L. 14); 4 ej. (L. 15); 1 ej. (L. 31); 7 ej. (L. 36).

\section{Herotilapia multispinosa (Günther)}

Heros multispinosus Günther, 1869: 453, (descripción original; Lago Managua; Guatemala).

Cicblasoma multispinosum Jordan \& Evermann, 1896: 1525, (descripción; Lago Mana. gua; Guatemala).

Herotilapia multispinosa Meek, 1907: 131, (descripción; Lagos Managua y Nicaragua); Regan, 1908: 31, (enumerado; Lago Managua).

La siguiente descripción se basa en diez ejemplares; cinco de 66 a 87 mm (L. 36); tres de 77 a $85 \mathrm{~mm}$ (L. 14); uno de $51 \mathrm{~mm}$ (L. 28); uno de 57 mm (L. 1).

Color oscuro con siete barras anchas verticales, mucho más oscuras en el centro, formando una banda lateral; manchas redondas oscuras en la parte superior del opérculo; mancha vertical alargada en la base de la aleta caudal.

Cuerpo profundo y robusto; aletas anal y dorsal bajas; parte blanca de las aletas dorsal y anal llega casi al extremo de la aleta caudal, la cual es redondeada. Boca pequeña; el extremo del maxilar no alcanza la vertical trazada por la parte anterior de la órbita.

La base de las aletas impares cubierta de escamas; aletas pélvicas largas, sobrepasando el origen de la aleta anal.

D. XVII-XIX, 8-9; A. X-XI, 6-8; Pct. 12-14; escamas línea lateral 26-29; fila de escamas en la mejilla 3-4; filamentos branquiales 3-4, 7-9.

Longitud estándard 51-87 mm (72.7); distancia predorsal 404-425 (411); distancia preanal 583-620 (601); distancia prepélvica $389-412$ (399); profundidad del cuerpo 44--537 (506); parte más estrecha del pedúnculo caudal 157. 178 (167); longitud de la cabeza 334-368 (350); longitud del morro 117-143 (128); diámetro de la órbita 90-108 (97); distancia interorbital 130-158 (144); longitud del maxilar 88-102 (93); ancho suborbital 59-80 (69); longitud aleta dorsal 785-875 (827); longitud de la aleta anal 542-636 (582); longitud de la aleta pectoral 251-315(294); longitud de la aleta pelviana 294-368 (334). base de la aleta dorsal 610-667 (633); base de la aleta anal 376-419 (390). 


\section{Familia ELEOTRIDAE}

(Fig. 14)

\section{Gobiomorus dormitor Lacépède}

Gobiomorus dormitor Lacépède, 1800: 583, (descripción original; Martinica, de un di bujo por Plumier).

Eleotris longiceps Günther, 1864: 151, (descripción original; Lago Nicaragua): Günther, 1869: 440, (descripción; Lago Nicaragua).

Pbilypnus longiceps Gill \& Bransford, 1877: 181, (enumerado; Lago Nicaragua).

Pbilypaus dormitor Jordan \& Evermann, 1896: 2194, (descripción, referencias; Lago Ni caragua); Meek, 1907: 131, (descripción; Lagos Managua y Nicaragua).

Pbilypnus dormitator Regan, 1908: 5, (descripción, referencias; Lago Nicaragua).

Esta especie es muy abundante en los lagos y muy buena como almiento. La siguiente descripción se basa en veinte ejemplares: dos de 175 y 228 mm (L. 1); ocho de 71 a $273 \mathrm{~mm}$ (L. 3); uno de $52 \mathrm{~mm}$ (L. 5); tres de 44 a $56 \mathrm{~mm}$ (L. 12); dos de 167 y $170 \mathrm{~mm}$ (L. 15); dos de 68 y $72 \mathrm{~mm}$ (L. 28); dos de 59 y $90 \mathrm{~mm}$ (L. 36).

Cuerpo veteado de castaño oscuro; banda oscura irregular, muy marcada en los ejemplares jóvenes, desde el opérculo hasta la base de la aleta caudal; aletas veteadas con manchas de color castaño. Los ejemplares se vuelven más oscuros con la edad, los más grandes son casi negros. La boca muy grande; la mandíbula inferior es la más larga.

D. VI-I, 10; A. I, 10; Pct. 16; escamas línea lateral 56-65; filamentos branquiales $3-5,9-13$.

Longitud estándard 44-272 mm (167); distancia predorsal 393-434 (415); distancia preanal 580-676 (638); distancia prepélvica 322-365 (349); profundidad del cuerpo 154-196 (179); parte más estrecha del pedúnculo caudal 94-114 (105); longitud de la cabeza 322-370 (339); longitud del morro 87116 (104); diámetro de la órbita 40-77 (60); distancia interorbital 54-77 (63); longitud del maxilar 107-165 (139); base de la aleta anal 119-139 (131); longitud de la aleta pectoral 182-220 (197); distancia de la punta del morro al origen de la segunda aleta dorsal 429-614 (583).

\section{Familia SYNBRANCHIDAE}

(Fig. 15)

\section{Synbrancbus marmoratus Bloch}

Synbranchus marmoratus Bloch, 1795: 87, (descripción original; Brasil).

Esta especie se encuentra desde México hasta el Brasil y no había sido 
reportada de Nicaragua. Fue examinado un ejemplar de unos $400 \mathrm{~mm}$ de largo, pescado por un grupo de alumnos del colegio.

\section{AGRADECIMIENTOS}

Agradezco al profesor L. R. Rivas, su apoyo, ciencia y aliento sin las cuales este trabajo y la expedición colectora hubieran sido imposibles.

Estoy especialmente endeudado con el Dr. Enrique Chamorro, en esa fecha Ministro de Agricultura de Nicaragua, que me dio toda clase de facilidades y ayuda financiera y que me proporcionó los servicios de Armando T. García, un hábil conductor $y$ asistente. Agradezco también el uso del laboratorio ictiológico y la biblioteca del departamento de Zoología de la Universidad de Miami por el equipo y las facilidades proporcionadas para mi trabajo.

El reverendo padre A. Aranguren, Rector de la Comunidad del Colegio Centro América y esta Comunidad me brindaron hospedaje y financiación para la expedición. Enrique José Chamorro, Armando Vega y Alfonso ArgüeIlo, así como varias personas de Granada, me acompañaron en varias ocasiones en mis expediciones. La Comunidad de Jesuitas de Mateare, especialmente el P. Artabe, me acogieron con su singular hospitalidad y me obsequiaron una valiosa colección de mapas de Nicaragua. A todas estas personas e instituciones mis sinceros agradecimientos.

\section{RESUMEN}

Para realizar este trabajo se recolectaron más de 10,000 peces en las costas de los grandes lagos de Nicaragua y ríos tributarios. Los ejemplares fueron clasificados utilizando las facilidades y la biblioteca de la Universidad de Miami.

Los peces clasificados se distribuyen en 16 familias, 27 géneros y 45 especies.

En el presente trabajo figuran claves para las familias, géneros y especies. En la descripción se han omitido, por conocidas, las referencias y caracteres de las familias y géneros.

En la descripción de cada especie figuran las referencias, que se ha pro. curado que sean lo más completas posible, generalmente una breve discusión sobre los caracteres de los ejemplares, posibles sinonimias y algún dato sobre su comportamiento. Termina la descripción de cada especie con una serie de medidas, siempre que ha sido posible, sobre una serie ontogenética de unos 20 ejemplares. Estas medidas expresadas en tantos por mil de la longitud total y en $\mathrm{mm}$, espero sean útiles a otros ictiólogos en la identificación de las citadas especies y discusión sobre posibles variaciones geográficas. 


\section{SUMMARY}

Sixteen families, 27 genera and 45 species of freshwater fishes of the Lakes basin of Nicaragua are listed, on the basis of some 10,000 specimens studied. Keys are given to the families, genera and species, with brief discussions of the characters of each species, partial synonymy and measurements. The latter, whenever possible, are based on ontogenic series of some 20 specimens, and are given in millimeters or as parts per thousand of the total length.

\section{ADDENDUM}

Las cinco especies descritas a continuación fueron encontradas después de haber escrito el presente trabajo. Se trata de especies ya conocidas y descritas en otras naciones de Centro América; por esta razón y también por falta de tiempo y medios materiales su descripción es breve y casi reducida a un nuevo record.

\section{Familia POECILIDAE}

\section{Poeciliopsis gracilis (Heckel)}

La especie ha sido descrita en México, Guatemala y Honduras. Nuestros ejemplares fueron pescados en el Lago Managua en un pequeño arroyo que corre en la finca Mateare, situada en la base de la península del mismo nombre. Fueron enviados para su identificación a Miami al Profesor L. R. Rivas y al Dr. R. R. Miller de Michigan.

Los ejemplares se ajustan en todo a la descripción de Rosen y BAILEY (45) para esta especie. Presentan la línea negra lateral dividida en varias manchas. Algunos ejemplares apenas presentaban una señal de esta línea. En todos los ejemplares aparece muy marcada la línea negra en el borde inferior del pedúnculo caudal.

\section{Belonesox belizanus Kner}

Belonesox belizanus Kner, 1860: 419-422 (descripción original; Belice, Honduras Británica )

No parecen ser muy abundantes en los lagos. Los pescadores del Lago Nicaragua los llaman "ulumina gaspara", por considerarlas como un término medio entre las uluminas (Mollienesia) y los gaspares (Lepisosteus).

Nuestros ejemplares fueron pescados en la costa del Lago Nicaragua en la localidad de Los Cocos, cerca del desagüe del Río Tipitapa.

Son doce ejemplares, seis machos y seis hembras, todos de tamaño pequeño. La hembra más grande mide $95 \mathrm{~mm}$ y el macho más pequeño $67 \mathrm{~mm}$. 
El color es oliváceo, muy oscuro en el dorso y amarillo sucio en el vientre; brillo azul verdoso en la cabeza y centro del cuerpo. La especie no había sido descrita en Nicaragua.

\section{Familia CENTROPOMIDAE}

Son peces muy fáciles de identificar por sus dos aletas dorsales, de espinas fuertes. La cabeza es alargada y cóncava. Aleta caudal bifurcada; línea lateral muy marcada; vientre recto; escamas etenoides; mandíbula inferior prominente; dientes villiformes en bandas; borde posterior del pre-opérculo con grandes espinas en los ángulos; dos aletas dorsales bien separadas, la primera con 8 espinas de las cuales son más largas la tercera y la cuarta; aleta anal con tres espinas.

\section{Centropomus parallelus Poey}

Fueron examinados dos ejemplares, de 35 y $50 \mathrm{cms}$, pescados en las costas de las Isletas de Granada. Se usó para su identificación la clave de L. R. Rivas (The Florida fishes of the genus Centropomus. Quait. J. Fla. Acad. Sci., 5: 53-64, 1962).

Escamas a lo largo de la línea lateral 86-87; radios anales 6; radios pectorales 15. La distancia entre el ano y el origen de la aleta anal era aproximadamente 1.5 de la distancia entre el ano y la inserción de las aletas pelvianas. Comunmente conocido como "robalo", no parece ser un pez muy abundante en el lago. Se pesca con más frecuencia en las cercanías de San Carlos, lo que hace suponer que sube las aguas del río San Juan desde el mai.

\section{Familia CYPRINODONTIDAE}

\section{Rivulus isthmensis Garman}

Rivulus isthmonsis, Garman (The Cyprinodonts, Mem. Mus. Comp. Zool. 19, No 1, July, 1895: 140. Río San José, Costa Rica).

Fueron pescados dos ejemplares en un canalito que sirve para regar una plantación de bananos, en una finca al pie del Volcán Mombacho, Granada. El canal es poco profundo y está lleno de hojas y barro.

Dos ejemplares, macho y hembra, de color oliváceo con manchas algo más oscuras muy borrosas, el vientre de color más claro, las aletas sin manchas excepto en la hembra que presentaba una marcada mancha ocelada en la parte superior de la aleta caudal. Ambos presentaban una banda más clara en la parte inferior de dicha aleta. 


\section{Familia GYMNOTIDAE \\ Gymnotus carapo Linnaeus}

Fue examinado un ejemplar pequeño, regalo del señor Armando Vega, pescado en Chontales. Su color es castaño con manchas claras formando estriaciones inclinadas hacia la cola. La longitud total es de $128 \mathrm{~mm}$; longitud de la cabeza $14 \mathrm{~mm}$; distancia del morro a origen aleta anal $26 \mathrm{~mm}$. Se han examinado también ocho ejemplares algo más grandes traídos del Río Siuna pero no han sido medidos pues deseamos conservarlos vivos.

\section{REFERENCIAS}

1. AstorQui, I.

1967. Investigaciones de un Jesuita en aguas de Nicaragua. Rev. Conservadora del Pensamiento Centroamericano, 79: 65-76.

2. BeEbe, W., \& J. TEE-VAN

1941. Eastern Pacific expeditions of the New York Zoological Society. XXVIII. Fishes from the tropical eastern Pacific. (From Cedros Island, Lower California, south to the Galapagos Islands and northern Peru). Zoologica, 26: 245-284.

3. Bigelow, H., \& W. SCHROEDER

1948. Sharks, p. 59-576. In H. Bigelow \& W. Schroeder, Fishes of the western Nortb Atlantic, pt. 1. Sears Found. Mar. Res., New Haven, Conn.

4. Bigelow, H., \& W. SCHROEdeR

1953. Sawfishes, guitarfishes, skates and rays, p. 1-314. In H. Bigelow \& W. Schroeder, Fishes of the western North Atlantic, pt. 2. Sears Found. Mar. Res., New Haven, Conn.

5. Bigelow, H., \& W. SCHROEder

1961. Carcharbinus nicaraguensis, a synonym of the bull shark $C$. leucas. Copeid. 1961: 359.

6. BLOCH, M. E.

1795. Icthyologie, ou bistoire naturelle, générale et particulaire des poissons. Avet des figures enluminézs dessinées d'après Nature. 12 Vol. Fol. Berlín.

7. Boeseman, $M$.

1956. Fresh-water sawfishes and sharks in Netherland's New Guinea. Science, 123: 222-223.

8. BOHLKE, J. E.

1958. Studies on fishes of the family Characidae. 16. A new Hyphessobrycon from Costa Rica. Bull. Fla. State. Mus., 3: 173-178.

9. Cunier, G.

1819. Sur les poissons du sous-genre Hydrocyon, sur deux nouvelles espèces de Chalceus, sur trois nouvelles espèces de Serrasalmes, et sur l'Argentina glos. sodonta de Foorskahl, qui est l'Albula gonorbynchus de Bloch. Mem. Mus. Hist. Nat. Paris, 5: 351-379. 
10. Cuvier G., \& M. Valenciennes

1828-49. Histoire Naturelle des Poissons. 22 Vols. Paris.

11. DuRham, H. W.

1944. New volcanoes and new mountain ranges. Science, 100: 49-50.

12. EigenmanN, C. H.

1907. On further collections of fishes from Paraguay, Ann. Carnegie Mus,, 4: 110-157.

13. Eigenmann, C. $\mathrm{H}$.

1908. Preliminary descriptions of new genera and species of Tetragonopterid Characins (Zoological results of the Thayer Brazilian expedition). Bull. Mus. Comp. Zool., 52: 91-106.

14. Eigenmann, C. $\mathrm{H}$.

1910. Catalogue of fresh water fishes of tropical and south temperate America. Report of the Princeton University Expedition to Patagonia, 1896-1899, 3: 375-511.

15. Ergenmann, C. H., \& F. Ogle

1907. An annotated list of Characin fishes in the United States National Museum and the Museum of Indiana University, with descriptions of new species. Proc. LI. S. Nat. Mus., 33: 1-36.

16. Fowler, H. W.

1923. Fishes from Nicaragua. Proc. Acad. Nat. Sci. Phila., 75: 23-32.

17. GiLL, $T$.

1863. (1864) Descriptive enumeration of a collection of fishes from the western coast of Central America presented to the Smithsonian Institution by Capt. John Dow. Proc. Acad. Nat. Sci. Pbila., 15: 162-174.

18. Gill, T., \& J. F. Bransford

1877. Synopsis of the fishes of lake Nicaragua. Proc. Acad. Nat. Sci. Pbila., 29: 175-191.

19. GÜNTHer, $\mathbf{A}$.

1860. Third list of cold-blooded vertebrata collected by Mr. Fraser in Ecuador. Proc. Zool. Soc. Lond., 1860: 233-240.

20. GÜNTHER, $A$.

1862. Catalogue of the Acanthopterygii Pharyngognathi and Anacanthini in the collection of the Bristish Museum. Cat. Fish. Brit. Mus., 4: 1-XII, 1-534

21. GÜNTHER, A.

1864. Report of a colection of fishes made by Messrs. Dow, Godman and Salvin, in Guatemala. Proc. Zool. Soc. Lond., 1864: 144-154.

22. GÜNTher, $A$.

1866. Memoir on the fishes of the States of Central America collected by Messrs. Salvin and Godman and Cpt. Dow. Proc. Zool. Soc. Lond., 1866: 600.604. 
23. GÜNTHER, A.

1869. An account of the fishes of the States of Central America based on collections made by Cpt. J. M. Dow, F. Godman, Esq. and O. Salvin, Esq. Trans. Zool. Soc. Lond., 6: 377-494.

24. HECKEL, J.

1840. Johann Natterers neue Flussfische Brasiliens nach den Beobachtungen und Mittheilungen des Entdeckers beschrieben. Ann. Wien Mus., 2: 325-471.

25. HILdEBRAND, S. F.

1925. Fishes of the Republic of El Salvador, Central America. U. S. Bull. Bur. Fish., 41: 238-287.

26. Hildebrand, S. F.

1938. A new catalogue of the fresh water fishes of Panama. Field Mus. Nat. Hist. Zool. Ser., 22: 219-359.

27. JORDAN, D. S.

1887. A preliminary list of the fishes of the West Indies. Proc. U. S. Nat. Nat. Mus., 1886, 9: 554-608.

28. JORDAN, D. S., \& B. W. EVERMANN

1896-1900. The fishes of North and Middle America. Bull. U. S. Nat. Mus., N 47, 4 vols.

29. Jordan, D. S., B. W. Evermann, \& H. W. Clark

1930. Check list of the fisbes and fishlike vertebrates of North and Middle America north of the northern boundary of Venezuela and Colombia. Rep. U. S. Comm. Fish., 1928 app. $X$, iv $+670 \mathrm{pp}$.

30. LACËPEDE, B. G.

1799-1804. Histoire Naturelle des Poissons. Paris. 15 Vols.

31. LATHAM. J.

1794. An essay on the various species of sawfish. Trans. Linn. Soc. Lond. (Zool.), 2: 273.282 .

32. LUTKEN, C.

1879-80. Smaa Bidrag Til Selachiernes Naturhistorie. Vidensk. Meddel. Dansk Naturbist. Foren. Kjobenhavn, 45-68.

33. MARden, L.

1944. A land of lakes and volcanoes. Nat. Geogr. Mag., 86: 161-184.

34. MEEK, S. E.

1908. Notes on fresh water fishes from Mexico and Central America. Publ. Field. Columbian Mus. 1907, 7: 133-157.

35. MEEK, S. E.

1912. New species of fishes from Costa Rica. Field Mus. Nat. Hist., 10: 69-75.

36. MeEK, S. E.

1914. An annotated list of fishes known to occur in the fresh waters of Costa Rica. Field Mus. Nat. Hist., 10: 101-134. 
37. Meek S. E., \& S. F. Hildebrand

1916. Fishes of the fresh waters of Panama. Field. Mus. Nat. Hist., 10: 217-374

38. MEeK S. E., \& S. F. HILDEBRAND

1923-1928. The marine fishes of Panama. Field Mus. Nat. Hist. Vol. 15 pt. 1. 1923;

pt. 3. 1928.

39. Miller, R. R.

1950. A review of the American Clupeid fishes of the genus Dorosomat. Proc. $U$. S. Nat. Mus, , 100: 387.410 .

40. Miller, R. R.

1954. The southern limit of the gar-fishes. Family Lepisosteidae. Copeia, 1954: $230-231$.

41. Muller, J., \& J. Henle

1841. Systematische Beschreibung der Plagiostomen. Berlin, 1838-41. XXII. 200 pp. 60 pls. fol.

42. Norman, J. R., \& F. C. Fraser

1937. Giant fisbes, whales and dolpbins. London, $361 \mathrm{pp}$.

43. REgAN, C. T.

1905. A revision of the fishes of the American genus Cicblosoma and of the allied genera. Ann. Mag. Nat. Hist., Ser. 7, 16: 60-77; 225-243; 316-340; 432. 445.

44. RrGAN, C. T.

1908. A collection of fresh water fishes made by Mr. C. F. Underwood in Costa Rica. Ann. Mag. Nat. Hist. Ser. 8, 2: 455.464.

45. Rosen, D. E., \& R. M. BAiley

1963. The Poecilid Fishes (Cyprinodontiformes), their structure, zoogeography, and systematics. Bull. Amcr. Mus. Nat. Hist., 126: 1-176.

46. SMITH, H. W.

1936. The retention and physiological role of urea in the Elasmobranchii. Biol. Rev: Cambridge, 11: 49.82.

47. Steindachner, F.

1869. Ichthyologische Notizen VIII. Sitzb. Ak. Wien, 1869: 120-139.

48. Thorson, T. B., D. E. Watson, \& C. M. COWAN

1966. The status of the freshwater shark of Lake Nicaragua. Copeia, 1966: 385402.

49. Zoppis-Bracci, L., * D. DEL GIUdice

1958. Geología de la costa del Pacífico de Nicaragua. Bol. Sent. Geol. Nacl. Nicaragua, 2: 25.68 . 